\title{
GRAIN GROWTH IN THE CIRCUMSTELLAR DISKS OF THE YOUNG STARS CY Tau AND DoAr 25
}

\author{
Laura M. Pérez ${ }^{1,17}$, Claire J. Chandler ${ }^{1}$, Andrea Isella ${ }^{2}$, John M. Carpenter ${ }^{3}$, Sean M. Andrews ${ }^{4}$, Nuria Calvet ${ }^{5}$, \\ Stuartt A. Corder ${ }^{6}$, Adam T. Deller ${ }^{7}$, Cornelis P. Dullemond ${ }^{8}$, Jane S. Greaves ${ }^{9}$, Robert J. Harris ${ }^{10}$, \\ Thomas Henning ${ }^{11}$, Woojin Kwon ${ }^{12}$, Joseph Lazio ${ }^{13}$, Hendrik Linz ${ }^{11}$, Lee G. Mundy ${ }^{14}$, Luca Ricci ${ }^{4}$, Anneila I. Sargent ${ }^{3}$, \\ Shaye Storm $^{14}$, Marco Tazzari ${ }^{15}$, Leonardo Testi ${ }^{15,16}$, and David J. Wilner ${ }^{4}$ \\ ${ }^{1}$ National Radio Astronomy Observatory, P.O. Box O, Socorro, NM 87801, USA \\ ${ }^{2}$ Rice University, 6100 Main Street, Houston, TX 77005, USA \\ ${ }^{3}$ California Institute of Technology, 1200 East California Blvd, Pasadena, CA 91125, USA \\ ${ }^{4}$ Harvard-Smithsonian Center for Astrophysics, 60 Garden Street, Cambridge, MA 02138, USA \\ ${ }^{5}$ University of Michigan, 830 Dennison Building, 500 Church Street, Ann Arbor, MI 48109, USA \\ ${ }^{6}$ Joint ALMA Observatory, Av. Alonso de Córdova 3107, Vitacura, Santiago, Chile \\ ${ }^{7}$ The Netherlands Institute for Radio Astronomy (ASTRON), 7990-AA Dwingeloo, The Netherlands \\ ${ }^{8}$ Heidelberg University, Center for Astronomy, Albert Ueberle Str 2, Heidelberg, Germany \\ ${ }^{9}$ University of St. Andrews, Physics and Astronomy, North Haugh, St. Andrews KY16 9SS, UK \\ ${ }^{10}$ University of Illinois, 1002 West Green St., Urbana, IL 61801, USA \\ ${ }^{11}$ Max-Planck-Institut für Astronomie, Königstuhl 17, D-69117 Heidelberg, Germany \\ ${ }^{12}$ Korea Astronomy and Space Science Institute, 776 Daedeok-daero, Yuseong-gu, Daejeon 34055, Korea \\ 13 Jet Propulsion Laboratory, California Institute of Technology, 4800 Oak Grove Dr, Pasadena, CA 91106, USA \\ ${ }^{14}$ University of Maryland, College Park, MD 20742, USA \\ ${ }^{15}$ European Southern Observatory, Karl Schwarzschild str. 2, D-85748 Garching, Germany \\ ${ }^{16}$ INAF-Osservatorio Astrofisico di Arcetri, Largo E. Fermi 5, I-50125 Firenze, Italy \\ Received 2015 May 4; accepted 2015 September 24; published 2015 October 27
}

\begin{abstract}
We present new results from the Disks@EVLA program for two young stars: CY Tau and DoAr 25. We trace continuum emission arising from their circusmtellar disks from spatially resolved observations, down to tens of AU scales, at $\lambda=0.9,2.8,8.0,9.8 \mathrm{~mm}$ for DoAr 25 and at $\lambda=1.3,2.8,7.1 \mathrm{~mm}$ for CY Tau. Additionally, we constrain the amount of emission whose origin is different from thermal dust emission from $5 \mathrm{~cm}$ observations. Directly from interferometric data, we find that observations at $7 \mathrm{~mm}$ and $1 \mathrm{~cm}$ trace emission from a compact disk while millimeter-wave observations trace an extended disk structure. From a physical disk model, where we characterize the disk structure of CY Tau and DoAr 25 at wavelengths shorter than $5 \mathrm{~cm}$, we find that (1) dust continuum emission is optically thin at the observed wavelengths and over the spatial scales studied, (2) a constant value of the dust opacity is not warranted by our observations, and (3) a high-significance radial gradient of the dust opacity spectral index, $\beta$, is consistent with the observed dust emission in both disks, with low- $\beta$ in the inner disk and high- $\beta$ in the outer disk. Assuming that changes in dust properties arise solely due to changes in the maximum particle size $\left(a_{\max }\right)$, we constrain radial variations of $a_{\max }$ in both disks, from cm-sized particles in the inner disk $(R<40 \mathrm{AU})$ to millimeter sizes in the outer disk $(R>80 \mathrm{AU})$. These observational constraints agree with theoretical predictions of the radial-drift barrier, however, fragmentation of dust grains could explain our $a_{\max }(R)$ constraints if these disks have lower turbulence and/or if dust can survive high-velocity collisions.
\end{abstract}

Key words: protoplanetary disks - radio continuum: planetary systems - stars: formation stars: individual (CY Tau, DoAr 25)

\section{INTRODUCTION}

The process of planet formation requires that small dust grains in the interstellar medium (ISM) undergo a dramatic transformation, growing by more than 12 orders of magnitude in order to form terrestrial planets and the cores of gas and ice giants. As a very first step, these $\mu \mathrm{m}$-sized ISM dust grains must increase their size and become macroscopic (Testi et al. 2014), a process that alters the optical properties of the dust grains considerably: as grains reach millimeter or larger sizes, the absolute value of the dust opacity, $\kappa_{\lambda}$, decreases, and at the same time the power-law spectral index of the dust opacity, $\beta$ (where $\kappa_{\lambda} \propto \lambda^{-\beta}$ ), becomes smaller (e.g., Miyake \& Nakagawa 1993; Henning \& Stognienko 1996; Draine 2006). Since thermal dust emission at millimeter and centimeter wavelengths is (mostly) in the optically thin regime, the observed dust continuum emission will trace the

\footnotetext{
17 Jansky Fellow of the National Radio Astronomy Observatory.
}

bulk of the disk mass modulated by the dust opacity: $S_{\nu} \propto \kappa_{\lambda} \times \Sigma \times B_{\nu}(T)$, where $\Sigma$ is the dust mass surface density and $B_{\nu}(T)$ is the Planck function evaluated at the dust temperature $T$. For optically thin dust, warm enough to be in the Rayleigh-Jeans (R-J) regime $(h \nu \ll k T)$, it follows that $S_{\nu}$ $\propto \nu^{(\beta+2)}$. Thus, direct measurements of the dust emission spectrum (i.e., the spectral energy distribution, SED, at long wavelengths) can be used to derive the value of $\beta$, a method extensively employed in the literature (Beckwith \& Sargent 1991; Henning et al. 1995; Testi et al. 2001; Calvet et al. 2002; Testi et al. 2003; Natta \& Testi 2004; Andrews \& Williams 2005, 2007; Wilner et al. 2005; Rodmann et al. 2006; Lommen et al. 2009; Ricci et al. 2010a, 2010b, 2011; Ubach et al. 2012).

Disk-integrated measurements, from sub-mm to $\mathrm{cm}$ wavelengths, have shown that in most protoplanetary disks the value of $\beta$ tends to be lower than in the ISM (where $\beta_{\text {ISM }} \sim 1.5-2.0$, consistent with the presence of $\mu \mathrm{m}$-sized dust grains or smaller in the ISM, Li \& Draine 2001). 
Possible explanations for the low values of $\beta$ include the presence of regions of high optical depth, which will drive the dust emission spectrum to be close to $S_{\nu} \propto \nu^{2}$, and thus a low $\beta$ would be inferred (incorrectly). However, Ricci et al. (2012) showed this would be only plausible for the brightest and most massive disks. Different composition and/or porosity could affect the optical properties of dust grains (e.g., Henning \& Mutschke 1997; Semenov et al. 2003; Boudet et al. 2005; Kataoka et al. 2014), but for a sensible set of dust properties the most significant influence on the opacity spectral index arises from an increase in grain size (Draine 2006). Thus, the difference in the dust opacity spectral index of disks, as compared to the ISM, implies that growth of at least four orders of magnitude in size has taken place inside circumstellar disks (for a review see: Natta et al. 2007; Testi et al. 2014). But since most of these measurements are of the global disk properties, they provide limited knowledge of the evolution of the dust properties as a function of the distance to the central star (hereafter referred to as radius).

Theoretical analyses predict that the average dust grain size will change with radius, as dust grows and fragments while it is transported throughout the disk (Dullemond \& Dominik 2005; Birnstiel et al. 2010). A fundamental limiting factor to the largest grain size is that of the radial drift problem. While solids move at Keplerian velocities in the disk, the gaseous component moves at slightly sub-Keplerian speed due to gas pressure support. This velocity difference induces a drag in the large particles, which end up losing angular momentum and drifting radially to smaller and smaller orbits, until they are accreted onto the star and lost from the solid population (Weidenschilling 1977). This barrier will limit the maximum grain size allowed in a disk, with $\mathrm{mm}$ - and $\mathrm{cm}$-sized particles at tens of AU from the star drifting inwards in timescale shorter than the disk lifetime (Youdin 2010). Thus, measuring changes in the dust properties as a function of radius is essential to investigate the effects of this barrier in the grain size distribution of disks.

Only recently have measurements reached the required sensitivity and a sufficient lever arm in wavelength to characterize radial variations of the dust properties (Isella et al. 2010; Banzatti et al. 2011; Guilloteau et al. 2011; Pérez et al. 2012; Trotta et al. 2013; Andrews et al. 2014; Menu et al. 2014). With wavelength coverage that spanned an order of magnitude, from sub-mm to $\mathrm{cm}$ wavelengths, observational constraints in dust opacity were obtained for the disk around the young star AS 209 (Pérez et al. 2012). In this study, a gradient in the value of $\beta$ was found from $\beta<0.5$ at $\sim 20 \mathrm{AU}$ to $\beta>1.5$ at more than $\sim 80 \mathrm{AU}$, while a constant value of the dust opacity throughout the disk failed to reproduce these observations. Furthermore, these results seem to agree with a population of dust grains limited by radial drift. Here we explore further this issue, by studying how the dust properties vary with radius in the protoplanetary disks that surround the young stars CY Tau, located in the Taurus star-forming region at a distance of $\sim 140 \mathrm{pc}$ (Torres et al. 2007), and DoAr 25, located in the L1688 dark cloud at a distance of $\sim 125 \mathrm{pc}$ (Loinard et al. 2008; Mamajek 2008). DoAr $25^{18}$ and CY Tau are pre-main sequence stars of K5 and M1 spectral type, respectively. Both stars are quite young: CY Tau is $2-3 \mathrm{Myr}$ old (Bertout et al. 2007; Guilloteau et al. 2014), while DoAr 25

\footnotetext{
18 Also known as GY92 17, WSB 29, and WLY 1-34.
}

is about 4 Myr old (Andrews et al. 2009), and both present a significant emission excess over the stellar photosphere, from near-infrared to millimeter wavelengths (Olofsson et al. 2009; McClure et al. 2010). DoAr 25 has been imaged at submillimeter wavelengths with the Submillimeter Array (Andrews et al. 2008, 2009), and CY Tau was imaged at the 1.3 and $2.8 \mathrm{~mm}$ bands with the Plateau de Bure Interferometer (Guilloteau et al. 2011).

In this paper, we triple the number of circumstellar disks for which our multiwavelength analysis has been used to measure any radial changes in the dust properties, particularly in the dust opacity. The paper is structured as follows. First, we describe the observations and data calibration procedures in Section 2, and we present observational results from these data in Section 3. In Section 4 we discuss the model of the disk emission employed in this paper, followed by Section 5 where observational constraints derived from this modeling are presented. Finally, in Section 6 we discuss our results in the context of grain growth, comparing these observational constraints with the radial drift and fragmentation barriers for growth, as well as with other disks previously studied. Our findings and conclusions are presented in Section 7.

\section{OBSERVATIONS}

We obtained multi-wavelength observations of the continuum emission from DoAr 25 and CY Tau with three interferometers: the Combined Array for Research in Millimeter-wave Astronomy (CARMA), the Sub-Millimeter Array (SMA), and the Karl G. Jansky Very Large Array (VLA) as part of the Disks@EVLA collaboration. CY Tau was observed at four different wavelengths: $1.3 \mathrm{~mm}, 2.8 \mathrm{~mm}, 7.1 \mathrm{~mm}$, and $5.0 \mathrm{~cm}$, with the highest angular resolution being 0.107 (9.8 AU) at $7.1 \mathrm{~mm}$. DoAr 25 was observed at five different wavelengths: $0.9 \mathrm{~mm}, 2.8 \mathrm{~mm}, 8.0 \mathrm{~mm}, 9.8 \mathrm{~mm}$, and $5.0 \mathrm{~cm}$, with the highest angular resolution being 0 ". $10(12.5 \mathrm{AU})$ at $8.0 \mathrm{~mm}$. A summary of these observations and our data calibration procedure is presented below.

\subsection{CARMA Observations}

CY Tau and DoAr 25 observations at $2.8 \mathrm{~mm}(107 \mathrm{GHz})$, and CY Tau observations at $1.3 \mathrm{~mm}(230 \mathrm{GHz})$ were obtained with CARMA in the $\mathrm{A}, \mathrm{B}$, and $\mathrm{C}$ configurations, providing baseline lengths between 30 and $2000 \mathrm{~m}$ which cover spatial scales from $20^{\prime \prime}$ down to 0.13 at $2.8 \mathrm{~mm}$, and between $9^{\prime \prime}$ down to 0 ". 13 at $1.3 \mathrm{~mm}$. A complete observing log of these observations can be found in Table 1. Double-sideband single-polarization receivers were tuned to a frequency of $107 \mathrm{GHz}$ in the case of $2.8 \mathrm{~mm}$ observations, and $230 \mathrm{GHz}$ in the case of $1.3 \mathrm{~mm}$ observations. To optimize the continuum sensitivity, we configured all spectral windows in the correlator to the maximum possible bandwidth: 468.75-MHz-wide windows of 15 channels each, which provided $2.8 \mathrm{GHz}$ of bandwidth for observations before the correlator upgrade in 2009 December, and 487.5-MHz-wide windows of 39 channels each, which provided $7.8 \mathrm{GHz}$ of total bandwidth after the upgrade.

The observing sequence interleaved observations of a complex gain calibrator (generally 3 minutes in length) with the science target. In $\mathrm{C}$ configuration the calibrator-target cycle time was 12-15 minutes, while in $\mathrm{A}$ and $\mathrm{B}$ 
Table 1

Observing Log for CARMA Observations

\begin{tabular}{|c|c|c|c|}
\hline Target & UT Date & Configuration $^{\mathrm{a}}$ & Phase Calibrator \\
\hline \multicolumn{4}{|c|}{$\lambda=1.3 \mathrm{~mm}$} \\
\hline \multirow[t]{6}{*}{ CY Tau } & 2007 Nov 07 & $\mathrm{C}, 0.35 \mathrm{~km}$ & $\mathrm{~J} 0530+1331$ \\
\hline & 2009 Jan 04 & $\mathrm{~B}, 1.0 \mathrm{~km}$ & 3C 111 \\
\hline & 2009 Jan 05 & $\mathrm{~B}, 1.0 \mathrm{~km}$ & 3C 111 \\
\hline & 2010 Sep 16 & $\mathrm{D}, 0.25 \mathrm{~km}$ & $\mathrm{~J} 0336+3218$ \\
\hline & 2010 Sep 21 & $\mathrm{D}, 0.25 \mathrm{~km}$ & $3 \mathrm{C} 111, \mathrm{~J} 0336+3218$ \\
\hline & 2011 Dec 12 & $\mathrm{~A}, 2.0 \mathrm{~km}$ & $\mathrm{~J} 0336+3218$ \\
\hline \multicolumn{4}{|c|}{$\lambda=2.8 \mathrm{~mm}$} \\
\hline \multirow{5}{*}{ CY Tau } & 2008 Dec 11 & $\mathrm{~B}, 1.0 \mathrm{~km}$ & 3C 111 \\
\hline & 2010 Feb 15 & $\mathrm{~A}, 2.0 \mathrm{~km}$ & $3 \mathrm{C} 111, \mathrm{~J} 0336+3218$ \\
\hline & 2011 Dec 30 & $\mathrm{~B}, 1.0 \mathrm{~km}$ & 3C 111 \\
\hline & 2012 Feb 08 & $\mathrm{C}, 0.35 \mathrm{~km}$ & 3C 111 \\
\hline & 2012 Feb 19 & $\mathrm{C}, 0.35 \mathrm{~km}$ & 3C 111 \\
\hline \multirow[t]{8}{*}{ DoAr 25} & 2010 Jan 06 & $\mathrm{~B}, 1.0 \mathrm{~km}$ & J1625-2527 \\
\hline & 2010 Jan 09 & $\mathrm{~B}, 1.0 \mathrm{~km}$ & J1625-2527 \\
\hline & 2010 Nov 29 & $\mathrm{~A}, 2.0 \mathrm{~km}$ & J1625-2527 \\
\hline & 2010 Nov 30 & $\mathrm{~A}, 2.0 \mathrm{~km}$ & J1625-2527 \\
\hline & 2010 Dec 03 & $\mathrm{~A}, 2.0 \mathrm{~km}$ & J1625-2527 \\
\hline & 2010 Dec 12 & $\mathrm{~A}, 2.0 \mathrm{~km}$ & $\mathrm{~J} 1625-2527$ \\
\hline & 2011 Apr 25 & $\mathrm{C}, 0.35 \mathrm{~km}$ & J1625-2527 \\
\hline & 2012 Jan 19 & $\mathrm{C}, 0.35 \mathrm{~km}$ & J1625-2527 \\
\hline
\end{tabular}

Note.

${ }^{\text {a }}$ Configuration name followed by the maximum baseline of each configuration.

configuration it was 5-10 minutes in order to track better the tropospheric phase fluctuations. This observing sequence repeated throughout the track, usually from target rise to set. In addition, a strong calibrator was observed to measure the complex bandpass, along with either a planet (Uranus, Mars, or Neptune) or a secondary flux density calibrator (either $3 \mathrm{C} 273$ or $3 \mathrm{C} 84$ ) that is monitored by the observatory. The estimated fractional uncertainty in the absolute flux density scale is $\sim 15 \%$.

The CARMA data were calibrated using the Multichannel Image Reconstruction, Image Analysis and Display (MIRIAD) software package (Sault et al. 1995). Each observation was calibrated separately. Malfunctioning antennas, receivers, and/or spectral windows were flagged, and updated antenna position corrections and line-length system corrections were applied. Observations of DoAr 25 in the most extended (A and B) configurations used the CARMA Paired Antenna Calibration System (C-PACS) to monitor the tropospheric delay fluctuations on eight of the outermost stations of the array, using adjacent $3.5 \mathrm{~m}$ antennas equipped with $1 \mathrm{~cm}$ receivers. Phase corrections derived from the C-PACS system are applied during post-processing. A complete description of C-PACS is presented by Pérez et al. (2010) and Zauderer et al. (2014).

\subsection{VLA Observations}

\subsubsection{High Frequency}

CY Tau observations at $7.1 \mathrm{~mm}(42 \mathrm{GHz}$, Q-band) were made during $\mathrm{A}, \mathrm{B}$, and $\mathrm{C}$ configurations of the VLA, while DoAr 25 observations at 8.0 and $9.8 \mathrm{~mm} \quad(30.5 \mathrm{Ghz}$ and
Table 2

Observing Log for VLA Observations

\begin{tabular}{|c|c|c|c|}
\hline Target & UT Date & Configuration $^{a}$ & Phase Calibrator \\
\hline \multicolumn{4}{|c|}{$\lambda=7.1 \mathrm{~mm}(\mathrm{Q}$ band $)$} \\
\hline \multirow[t]{6}{*}{ CY Tau } & 2010 Nov 13 & $\mathrm{C}, 3.4 \mathrm{~km}$ & $\mathrm{~J} 0403+2600$ \\
\hline & 2011 Apr 05 & $\mathrm{~B}, 11.1 \mathrm{~km}$ & $\mathrm{~J} 0403+2600$ \\
\hline & 2012 Oct 22 & $\mathrm{~A}, 36.4 \mathrm{~km}$ & $\mathrm{~J} 0403+2600$ \\
\hline & 2012 Oct 26 & $\mathrm{~A}, 36.4 \mathrm{~km}$ & $\mathrm{~J} 0403+2600$ \\
\hline & 2012 Oct 27 & $\mathrm{~A}, 36.4 \mathrm{~km}$ & $\mathrm{~J} 0403+2600$ \\
\hline & 2012 Oct 28 & $\mathrm{~A}, 36.4 \mathrm{~km}$ & $\mathrm{~J} 0403+2600$ \\
\hline \multicolumn{4}{|c|}{$\lambda=8.0$ and $9.8 \mathrm{~mm}(\mathrm{Ka}$ band $)$} \\
\hline \multirow[t]{3}{*}{ DoAr 25} & 2011 Jan 23 & $\mathrm{CnB}, 11.1 \mathrm{~km}$ & $\mathrm{~J} 1625-2527$ \\
\hline & 2011 May 28 & $\mathrm{BnA}, 36.4 \mathrm{~km}$ & $\mathrm{~J} 1625-2527$ \\
\hline & 2011 Jun 15 & $\mathrm{~A}, 36.4 \mathrm{~km}$ & $\mathrm{~J} 1625-2527$ \\
\hline \multicolumn{4}{|c|}{$\lambda=5.0 \mathrm{~cm}(\mathrm{C}$ band $)$} \\
\hline CY Tau & 2011 Jul 23 & $\mathrm{~A}, 36.4 \mathrm{~km}$ & $\mathrm{~J} 0403+2600$ \\
\hline DoAr 25 & 2011 Jul 14 & $\mathrm{~A}, 36.4 \mathrm{~km}$ & $\mathrm{~J} 1625-2527$ \\
\hline
\end{tabular}

Note.

${ }^{\text {a }}$ Configuration name followed by the maximum baseline of each configuration.

$37.5 \mathrm{GHz}$, Ka-band) were made during $\mathrm{A}, \mathrm{BnA}$, and $\mathrm{CnB}$ configurations, ${ }^{19}$ providing baseline lengths for both targets between $60 \mathrm{~m}$ to $36 \mathrm{~km}$. These baseline lengths correspond to spatial scale coverage from about $24^{\prime \prime}$ down to $0 . " 04$ at $7.1 \mathrm{~mm}$, and from about $34^{\prime \prime}$ down to 0.06 at $9.8 \mathrm{~mm}$. These observations, summarized in Table 2, used dual-polarization receivers and two independently tunable basebands, where each baseband was configured to eight $128 \mathrm{MHz}$ spectral windows of 64 channels, to provide the maximum continuum bandwidth per baseband $(1 \mathrm{GHz})$. For the Q-band observations, the two basebands were centered at $7.1 \mathrm{~mm}(42.5 \mathrm{GHz})$ and $7.2 \mathrm{~mm}(41.5 \mathrm{GHz})$ providing $2 \mathrm{GHz}$ total bandwidth at $7.14 \mathrm{~mm}$, while for the Ka-band observations, the two $1 \mathrm{GHz}$ basebands were centered at $8.0 \mathrm{~mm}(37.5 \mathrm{GHz})$ and $9.8 \mathrm{~mm}$ $(30.5 \mathrm{GHz})$.

\subsubsection{Low Frequency}

To distinguish the amount of free-free or non-thermal contamination present at millimeter and centimeter wavelengths, CY Tau and DoAr 25 were observed at $5.0 \mathrm{~cm}$ $(6.0 \mathrm{GHz})$ in C-band. These low-frequency VLA observations were obtained during A configuration, with dualpolarization receivers and two independently tunable basebands, where each baseband was configured as for the high frequency observations with a total bandwidth per baseband of $1 \mathrm{GHz}$. The two basebands were centered at $6.2 \mathrm{~cm}$ $(4.8 \mathrm{GHz})$ and $4.1 \mathrm{~cm}(7.3 \mathrm{GHz})$, in order to be able to infer the spectral slope of the emission at centimeter wavelengths.

\footnotetext{
19 The hybrid $\mathrm{BnA}$ and $\mathrm{CnB}$ configurations comprise antennas on the east and west arms of the VLA moved to their locations for the more compact configuration, while the antennas on the north arm are moved to their locations for the more extended configuration, so that in projection, for southern sources, the synthesized beam is circularized.
} 


\subsubsection{VLA Data Acquisition and Calibration}

The observing sequence interleaved observations of a complex gain calibrator ( $45 \mathrm{~s}$ to a few minutes in length) with science target observations, whose duration depended on the array configuration and observing frequency: target-calibrator cycle times in extended configurations were 1.5-5 minutes at high frequencies, 5-10 minutes in compact configurations. The target-calibrator cycle time at C-band in A-configuration was 10-15 minutes. A strong calibrator was used to determine the bandpass shape. The absolute flux density calibration was determined from observations of a primary flux density calibrator (3C147 for CY Tau, 3C286 for DoAr 25). Models for the primary flux density calibrators are provided by the observatory. The estimated uncertainty in the absolute flux density scale is $5 \%$ at $\mathrm{C}$-band, and $10 \%$ at $\mathrm{Ka}$ and Q bands.

The VLA data calibration was performed using the CASA software package and a modified version of the VLA calibration pipeline. ${ }^{20}$ As with the CARMA observations, each observation was calibrated separately. Malfunctioning antennas, receivers, and/or spectral windows were flagged, as well as noisy channels at the edge of each spectral window. The first few seconds at the beginning of each target observation were also flagged, along with any radio frequency interference (especially an issue with the C-band data). Times of poor phase coherence, as measured on the gain calibrator scans, were also removed from the data. Because the data were acquired over an extended time period, corrections for source proper motion or other systematic position offsets were applied prior to combining. All VLA data presented here were obtained as part of the Disks@EVLA project (AC982).

\subsection{SMA Observations}

DoAr 25 observations at $0.9 \mathrm{~mm}(345 \mathrm{GHz})$ were obtained with the SMA between 2005 May and 2007 June; these observations have already been presented by Andrews et al. (2008, 2009). Three different array configurations (C, E, and $\mathrm{V}$ ) were used, providing baseline lengths between 8 and $500 \mathrm{~m}$, corresponding to spatial scales from about $22^{\prime \prime}$ down to 0 ". 3 . Double-sideband receivers were tuned to a local oscillator frequency of $340.755 \mathrm{GHz}$. Each sideband was configured to have 24 partially overlapping $104 \mathrm{MHz}$ chunks, for a total continuum bandwidth of $4 \mathrm{GHz}$. The observing sequence interleaved observations of a complex gain calibrator with science target observations twice as long. The source-calibrator cycle was 8 minutes for the extended configuration, and 15-20 minutes for compact configurations. Bandpass and flux density calibrators were selected from different planets and satellites (Uranus, Jupiter, Saturn, Titan, Callisto), as well as strong quasars (3C454.3, 3C279), depending on their availability and array configuration. The estimated uncertainty in the absolute flux density scale is $\sim 10 \%$. The data were flagged and calibrated with the IDL-based MIR software package.

\subsection{Averaging of Interferometric Data}

For each telescope we verified that the calibration from multiple observations obtained in different array configurations matched, to within the uncertainty in the absolute flux scale, by comparing the calibrated visibilities where they overlap in $u v$ space. The observations at $0.9,1.3,2.8,7.1,8.0$, and $9.8 \mathrm{~mm}$

\footnotetext{
${ }^{20}$ https://science.nrao.edu/facilities/vla/data-processing/pipeline
}

were then averaged over the observing bandwidth, as follows. In the case of the $0.9,1.3,2.8$, and $7 \mathrm{~mm}$ data, visibilities encompass a narrow range of frequencies over the entire bandwidth: $\Delta \lambda / \lambda \sim 0.01$ at $0.9 \mathrm{~mm}, \Delta \lambda / \lambda \sim 0.04$ at $1.3 \mathrm{~mm}$, $\Delta \lambda / \lambda \sim 0.08$ at $2.8 \mathrm{~mm}$, and $\Delta \lambda / \lambda \sim 0.05$ at $7.1 \mathrm{~mm}$. These data were therefore averaged into a single wideband channel. In the case of Ka-band VLA observations, the two 1-GHz wide basebands are separated by $\sim 7 \mathrm{GHz}$, so these data were averaged into two wideband channels: one centered at $30.5 \mathrm{GHz}(\lambda=9.8 \mathrm{~mm})$, the other centered at $37.5 \mathrm{GHz}$ $(\lambda=8.0 \mathrm{~mm})$. Calibrated observations from different array configurations were combined to generate a single visibility file for each wavelength analyzed, in order to compare these interferometric observations with physical disk models.

\section{OBSERVATIONAL RESULTS}

\section{1. $C Y$ Tau}

Synthesized maps at $1.3,2.8$, and $7.1 \mathrm{~mm}$ of the continuum emission from CY Tau are presented in Figure 1. Each map extends 2 ". $5 \times 2$ ". 5 , corresponding to $350 \mathrm{AU}$ at the adopted distance. At wavelengths of $1.3 \mathrm{~mm}, 7.1 \mathrm{~mm}$, and $5.0 \mathrm{~cm}$, natural weighting was used to maximize the sensitivity. At a wavelength of $2.8 \mathrm{~mm}$, a Briggs weighting scheme with a robust parameter of 0.5 was used in CASA, to optimize a combination of resolution and sensitivity. The resulting image properties and source photometry can be found in Table 3, from these measurements we infer an spectral index for CY Tau from 1.3 to $7.1 \mathrm{~mm}$ of $\alpha=2.6$.

The observations at $5 \mathrm{~cm}$ were used to estimate the contribution from processes other than thermal dust emission at $7.1 \mathrm{~mm}$ (e.g., chromospheric activity or thermal bremsstrahlung from photoevaporative disk winds driven by the central protostar; Mundy et al. 1993; Pascucci et al. 2011), which needs to be taken into account when modeling the dust emission from the disk. However, CY Tau is not detected at $5 \mathrm{~cm}$, thus we take the $3 \sigma$ upper limit of $20 \mu \mathrm{Jy}$ and estimate the maximum possible contamination at $7.1 \mathrm{~mm}$ by assuming optically thick free-free emission with spherical symmetry, for which the emission as a function of frequency, $\nu$, is proportional to $S_{\nu} \propto \nu^{0.6}$ (Reynolds 1986). The maximum contamination at $7.1 \mathrm{~mm}$ thus corresponds to $\sim 60 \mu \mathrm{Jy}$, which represents only $4 \%$ of the total emission at this wavelength. We note that the probable source of any such contamination will arise from very near the protostar, and would appear as an unresolved point source in the $7.1 \mathrm{~mm}$ data. Based on the $7.1 \mathrm{~mm}$ data themselves, using $u v$-distances $\geqslant 10 \mathrm{~km}$ (corresponding to spatial scales smaller than $\sim 0$ "' 14), we constrain any unresolved point source to have a flux density of $74 \pm$ $9 \mu \mathrm{Jy}$ (some of which could be dust emission), consistent with our estimate of the potential contamination extrapolated from $5 \mathrm{~cm}$.

\section{2. $\operatorname{DoAr} 25$}

Synthesized maps at $0.9,2.8,8.0$, and $9.8 \mathrm{~mm}$ of the continuum emission from CY Tau are presented in Figure 2. Each map extends 2 ". $8 \times 2$ ". 8 , corresponding to $350 \mathrm{AU}$ at the adopted distance. Natural weighting was used in the imaging at wavelengths of $8.0 \mathrm{~mm}, 9.8 \mathrm{~mm}$, and $5.0 \mathrm{~cm}$, to maximize the sensitivity. Briggs weighting with robust parameters of 0.7 and 0.3 were used for imaging at wavelengths of 0.9 and $2.8 \mathrm{~mm}$, respectively, to optimize the resolution and sensitivity at these 

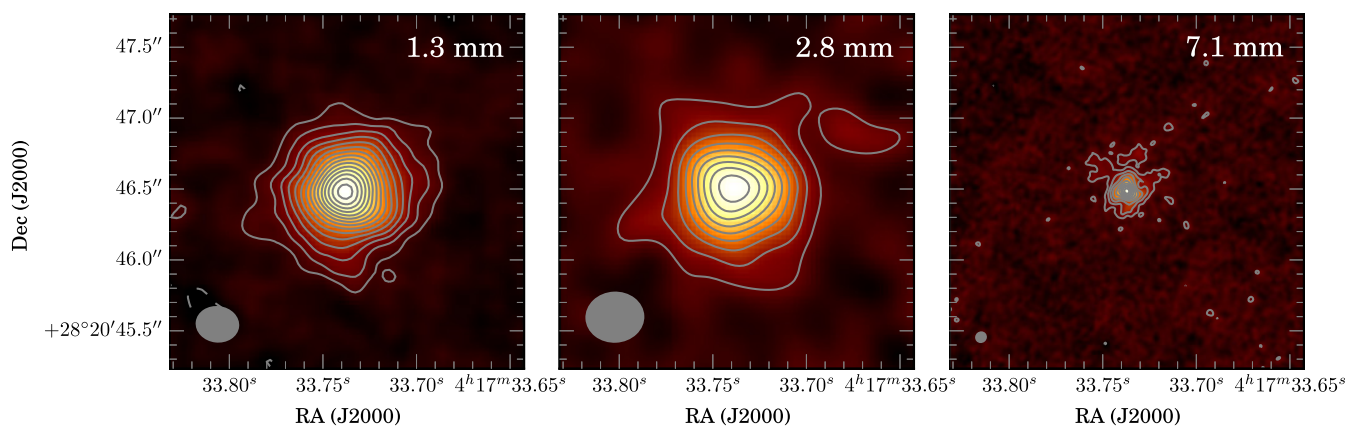

Figure 1. Aperture synthesis images of the continuum emission toward the young star CY Tau, observed at wavelengths of $1.3,2.8$, and $7.1 \mathrm{~mm}$. Each panel encompasses a 2 ". $5 \times 2$ ". 5 region ( $350 \mathrm{AU}$ in size at the adopted distance), with contours drawn at $3 \sigma$ intervals, where $\sigma$ is the rms noise level in each map (see Table 3).

Table 3

CY Tau Imaging and Photometry Results

\begin{tabular}{|c|c|c|c|c|c|}
\hline \multirow[b]{2}{*}{ Telescope } & \multirow[b]{2}{*}{$\begin{array}{c}\lambda \\
(\mathrm{mm})\end{array}$} & \multicolumn{3}{|c|}{ Image Properties } & \multirow{2}{*}{$\begin{array}{c}\text { Source Photometry }^{\mathrm{a}} \\
\begin{array}{c}\text { Flux Density } \\
(\mathrm{mJy})\end{array}\end{array}$} \\
\hline & & $\begin{array}{c}\text { Image rms Noise } \\
(\mu \mathrm{Jy} / \text { beam })\end{array}$ & Synthesized Beam & $\begin{array}{c}\text { Beam P.A. } \\
\left({ }^{\circ}\right)\end{array}$ & \\
\hline CARMA & 1.3 & 580 & $0 ! .29 \times 0 ! .24$ & 83.3 & $119 \pm 18$ \\
\hline CARMA & 2.8 & 280 & 0 ". $40 \times 0$ " 35 & -87.1 & $26.1 \pm 3.9$ \\
\hline VLA & 7.1 & 7.8 & $0 ! .07 \times 0 . " 07$ & -49.5 & $1.76 \pm 0.27$ \\
\hline VLA & 50 & 7.0 & 0 !" $62 \times 0$ "! 34 & -63.7 & $<0.020(3 \sigma)$ \\
\hline
\end{tabular}

Note.

${ }^{\mathrm{a}}$ Source photometry obtained from the measured flux density of CY Tau limited to $u v$-distances between 0 and $40 \mathrm{k} \lambda$, corresponding to the shortest $u v$-spacings we have sampled, except for $\lambda=50 \mathrm{~mm}$ which is not detected and thus we can only provide an upper limit. Note that the errors quoted include the uncertainty in the absolute flux density scale.
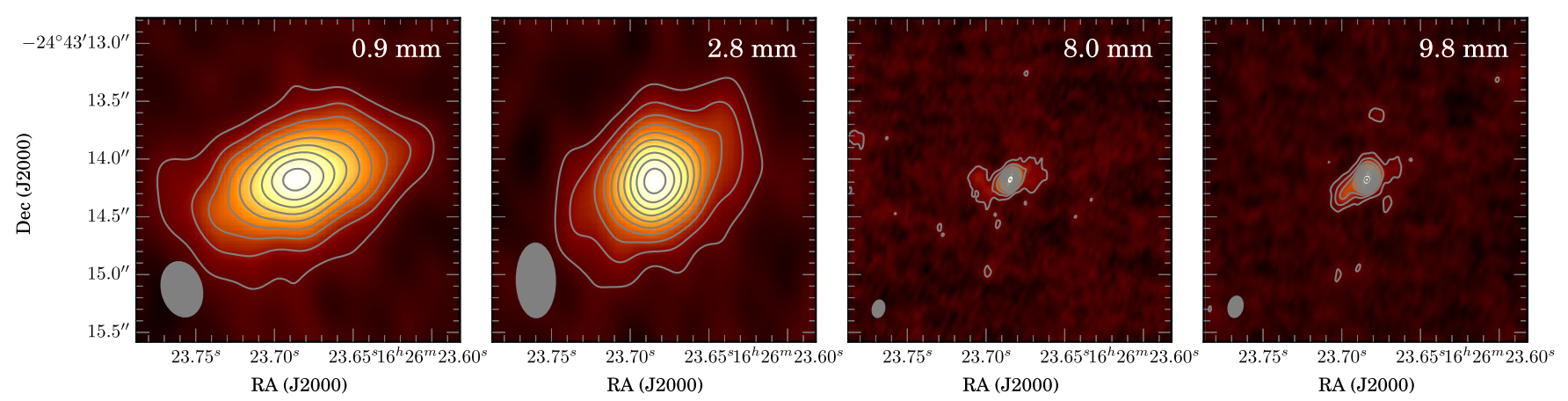

Figure 2. Aperture synthesis images of the continuum emission toward the young star DoAr 25, observed at wavelengths of 0.9, 2.8, 8.0, and 9.8 mm. Each panel encompasses a 2 ! $8 \times 2$ "! 7 region ( $350 \mathrm{AU}$ in size at the adopted distance), with contours drawn at $3 \sigma$ intervals, where $\sigma$ is the rms noise level in each map (see Table 4).

Table 4

DoAr 25 Imaging and Photometry Results

\begin{tabular}{|c|c|c|c|c|c|}
\hline \multirow[b]{2}{*}{ Telescope } & \multirow[b]{2}{*}{$\begin{array}{c}\lambda \\
(\mathrm{mm})\end{array}$} & \multicolumn{3}{|c|}{ Image Properties } & \multirow{2}{*}{$\begin{array}{c}\text { Source Photometry } \\
\text { Flux Density } \\
(\mathrm{mJy})\end{array}$} \\
\hline & & $\begin{array}{l}\text { Image rms Noise } \\
(\mu \mathrm{Jy} / \text { beam })\end{array}$ & Synthesized Beam & $\begin{array}{c}\text { Beam P.A. } \\
\left({ }^{\circ}\right)\end{array}$ & \\
\hline SMA & 0.9 & 3500 & $0 ! .48 \times 0$ ". 35 & 14.4 & $515 \pm 52$ \\
\hline CARMA & 2.8 & 270 & $0.64 \times 0 . .33$ & 0.9 & $28.1 \pm 4.2$ \\
\hline VLA & 8.0 & 11.0 & $0 " .15 \times 0$ " 10 & -13.2 & $1.17 \pm 0.13$ \\
\hline VLA & 9.8 & 8.0 & $0{ }^{\prime \prime} 18 \times 0{ }^{\prime \prime} 12$ & -13.3 & $0.66 \pm 0.07$ \\
\hline VLA & 50 & 5.9 & 0 ". $68 \times 0$ ×. 34 & -8.4 & $0.051 \pm 0.012$ \\
\hline
\end{tabular}

Note.

${ }^{\text {a }}$ Source photometry obtained from the measured flux density of CY Tau limited to $u v$-distances between 0 and $40 \mathrm{k} \lambda$, corresponding to the shortest $u v$-spacings we have sampled except for $\lambda=50 \mathrm{~mm}$, where the source is weak and unresolved and the fit is performed in the image domain. Note that the errors quoted include the uncertainty in the absolute flux density scale. 
wavelengths. The resulting image properties and source photometry can be found in Table 4, from these measurements we infer an spectral index for DoAr 25 from 0.9 to $9.8 \mathrm{~mm}$ of $\alpha=2.8$.

As for CY Tau, the observations at $5 \mathrm{~cm}$ from DoAr 25 were used to estimate the contribution from other than thermal dust emission at 8.0 and $9.8 \mathrm{~mm}$. In the case of DoAr 25, $\lambda=5 \mathrm{~cm}$ emission is detected at a level of $6 \sigma$ coincident with the star, with an integrated flux density of $50 \pm 13 \mu \mathrm{Jy}$. Assuming $S_{\nu} \propto$ $\nu^{0.6}$ results in an estimated contamination of $150 \pm 40 \mu \mathrm{Jy}$ at $8.0 \mathrm{~mm}$, and $133 \pm 35 \mu \mathrm{Jy}$ at $9.8 \mathrm{~mm}$, which corresponds to $17 \%$ and $24 \%$ of the integrated emission at these wavelengths. A lower level of contamination is derived based only in the 8.0 and $9.8 \mathrm{~mm}$ data with $u v$-distances $\geqslant 10 \mathrm{~km}$ (corresponding to spatial scales smaller than $\sim 0$ !" 17): an unresolved point source flux density of $68 \pm 24 \mu \mathrm{Jy}$ is present in the $8.0 \mathrm{~mm}$ observations, while a flux density of $65 \pm 16 \mu \mathrm{Jy}$ is constrained at $9.8 \mathrm{~mm}$. These estimates are consistent (within the errorbars) with the extrapolation from $5 \mathrm{~cm}$. However, we adopt the most conservative value (the highest possible contamination) in our analysis.

\subsection{Brightness Temperature and Optical Depth of the Emission}

The brightness temperature of the emission at $\mathrm{mm}$ and $\mathrm{cm}$ wavelengths can be useful to discriminate between optically thin and optically thick emission. For a medium at a physical temperature $T$ with no background radiation and an optical depth $\tau$, the brightness temperature $\left(T_{B}\right)$ of the emission at a particular wavelength $\lambda$ is related to $T$ as: $T_{B}(\lambda)=T\left(1-e^{-\tau_{\lambda}}\right)$ (see, e.g., Wilson et al. 2009). Thus, in the optically thick limit $(\tau \gg 1)$ the brightness temperature directly traces the temperature of the medium and is independent of wavelength: $T_{B}(\lambda)=T$, while in the optically thin limit $(\tau \ll 1)$ the brightness temperature will be much lower than the physical temperature of the medium: $T_{B}(\lambda)=\tau_{\lambda} T$ and will vary with wavelength according to the wavelength dependence of $\tau_{\lambda}$.

The data presented in Sections 3.1 and 3.2 include high frequency observations and emission from the cold outer disk of CY Tau and DoAr 25, thus the R-J limit of $h \nu \ll k T$ might not be appropriate even at $\mathrm{cm}$ wavelengths. For this reason we calculate the brightness temperature of the emission using the full Planck function without this approximation. Figures 3 and 4 present azimuthally averaged radial $T_{B}$ profiles for both disks at each observed wavelength, with the shaded region representing the $1 \sigma$ constraint derived from the statistical uncertainty in the images. For a proper comparison, we convolved all observations to the same angular resolution (i.e., the lowest spatial resolution, which corresponds to the resolution of the $2.8 \mathrm{~mm}$ data). In addition, we subtracted an unresolved point-source component for the long-wavelength observations at $7.1 \mathrm{~mm}$ (CY Tau) and at 8.0 and $9.8 \mathrm{~mm}$ (DoAr 25), whose flux density is given by the amount of contamination from non-dust emission described in Sections 3.1 and 3.2.

The observed brightness temperature profiles in Figures 3 and 4 decline with increasing distance from the star in both disks, becoming successively fainter as the wavelength of the emission is increased. If the observed emission were optically thick, the $T_{B}$ profiles would directly trace the physical temperature of the disk. For a vertically isothermal disk in

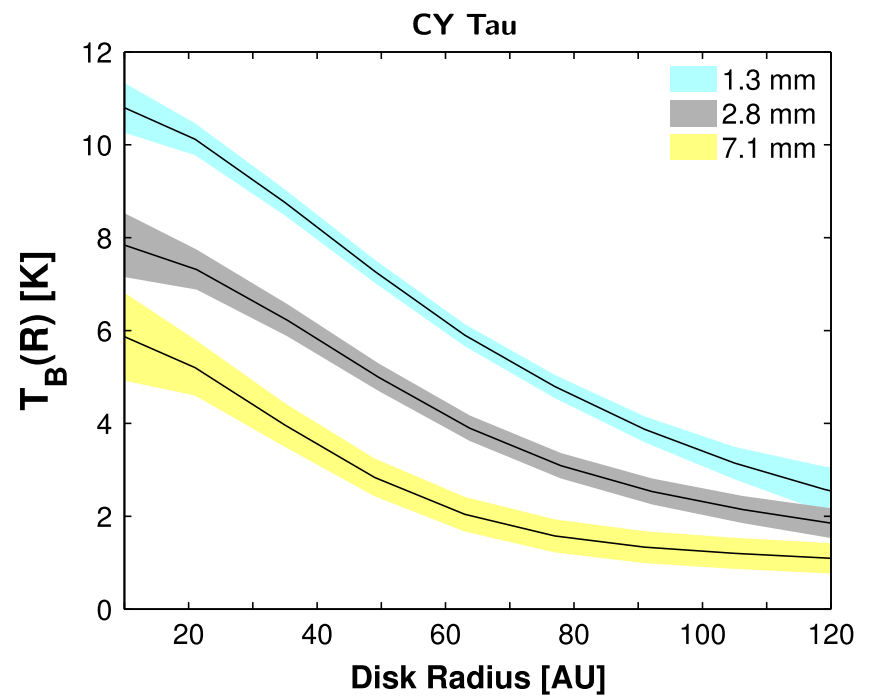

Figure 3. Observed brightness temperature of the emission from our multiwavelength observations of CY Tau, as a function of the radial distance to the central star. These profiles are being compared at the same angular resolution, corresponding to the $2.8 \mathrm{~mm}$ observations $(0$ "! $4,56 \mathrm{AU})$.

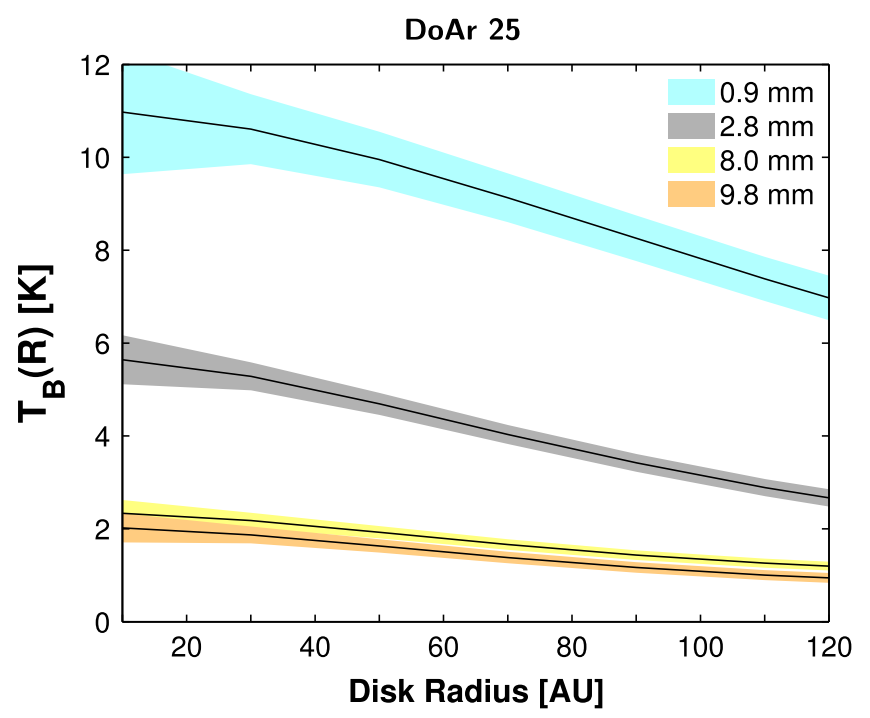

Figure 4. Observed brightness temperature of the emission from our multiwavelength observations of DoAr 25, as a function of the radial distance to the central star. These profiles are being compared at the same angular resolution, corresponding to the $2.8 \mathrm{~mm}$ observations $(0$." $64,80 \mathrm{AU})$.

this limit, the observed brightness temperature should then be the same at all wavelengths. For both DoAr 25 and CY Tau we observe a successively fainter $T_{B}$ profile with increasing wavelength, suggesting that for all but the shortest wavelength, the observed emission is consistent with being optically thin. Furthermore, in Section 5 we find that, for a flared disk in hydrostatic equilibrium, the midplane temperature is a factor of several higher than the observed brightness temperature of CY Tau and DoAr 25 at all disk radii and at all wavelengths, thus indicating that the observed emission is optically thin rather than optically thick (see section Section 4 for details of the modeling and Section 5.1 for the resulting midplane temperature profiles).

A colder midplane temperature could be found in the extreme case of a thin flat disk in the presence of no interstellar radiation. This configuration results in the coldest midplane 


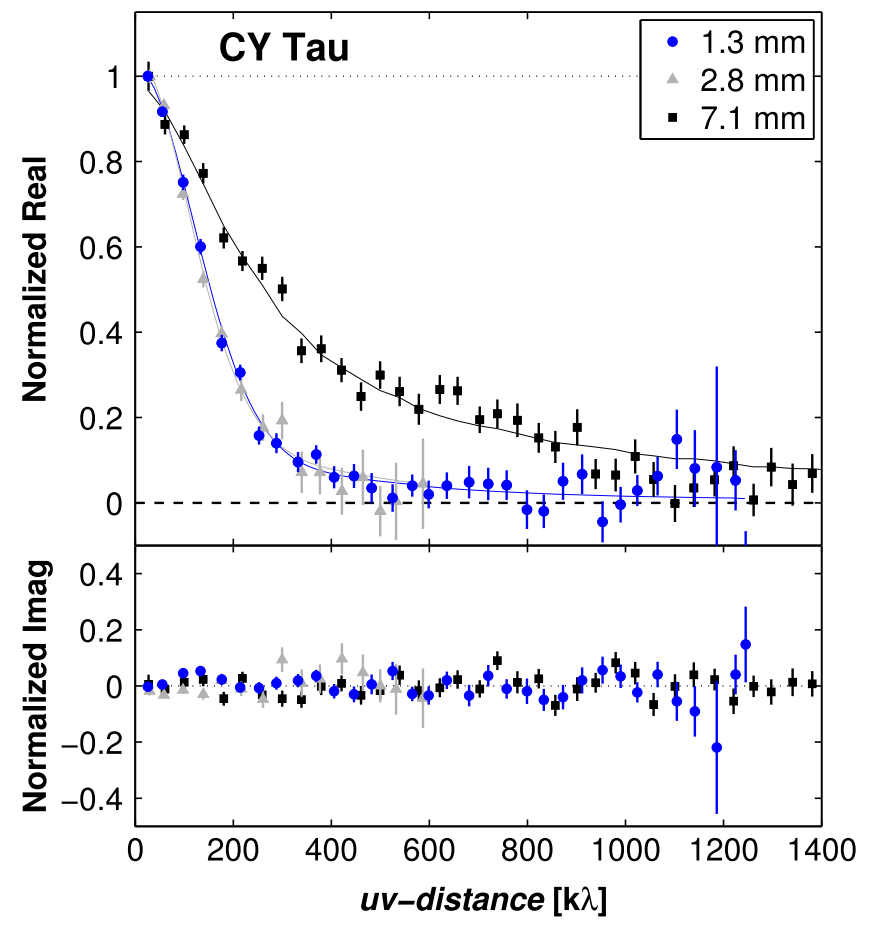

Figure 5. Normalized real and imaginary part of the correlated emission from CY Tau as a function of spatial frequency ( $u v$-distance). The visibilities have been de-projected using a position angle of $63^{\circ}$ and inclination of $28^{\circ}$, derived from molecular line observations (Guilloteau et al. 2011). Each bin has a width of $40 \mathrm{k} \lambda$, and has been normalized by the measured flux density at $0-40 \mathrm{k} \lambda$. Filled circles and error bars of different color correspond to correlated real and imaginary part of the emission observed at different wavelengths $(1.3 \mathrm{~mm}$ : blue circle, $2.8 \mathrm{~mm}$ : gray triangle, $7.1 \mathrm{~mm}$ : black square). The continuous lines correspond to the best-fit disk emission model at each wavelength (see modeling in Section 5.1).

disk possible, since the opening angle of a thin disk is much smaller than for a flared disk, making the stellar heating less efficient. Note that a more settled disk interpretation is favored in the analysis of unresolved observations of CY Tau, as the SED from mid-infrared to far-infrared wavelengths is quite steep for this object (see, e.g., Robitaille et al. 2007). In the case of DoAr 25, Spitzer IRS spectra reveal a flat spectrum between 10 and $30 \mu \mathrm{m}$ consistent with a flared disk structure (Olofsson et al. 2009; McClure et al. 2010). Thus, we only consider the thin disk case for CY Tau. We find a midplane temperature of $\sim 14 \mathrm{~K}$ at $10 \mathrm{AU}$, decreasing to a lower limit defined by interstellar radiation and cosmic ray heating (assumed to be $\sim 8 \mathrm{~K}$ ) at radii of $30 \mathrm{AU}$ and greater. Only for this extreme case is the observed $T_{B}$ profile of CY Tau consistent with the physical temperature of the disk midplane, and this is only for the shortest wavelength observations at $1.3 \mathrm{~mm}$ at radii $\sim 30-50 \mathrm{AU}$. At longer wavelengths (and at larger radii at $1.3 \mathrm{~mm}$ ) the brightness temperature is still below this cold midplane case, which indicates that the optical depth of the emission is less than 1 , even in this extreme case. Consequently, we will assume in the following analysis that the emission at $1.3 \mathrm{~mm}$ from CY Tau is optically thin, although there is a possibility of higher optical depth at $1.3 \mathrm{~mm}$ if the disk structure corresponds to a flat thin disk for this object.

\subsection{De-projected Visibility Profiles}

In Figures 5 and 6, we present the real and imaginary part of the visibility as a function of $u v$-distance (hereafter referred to

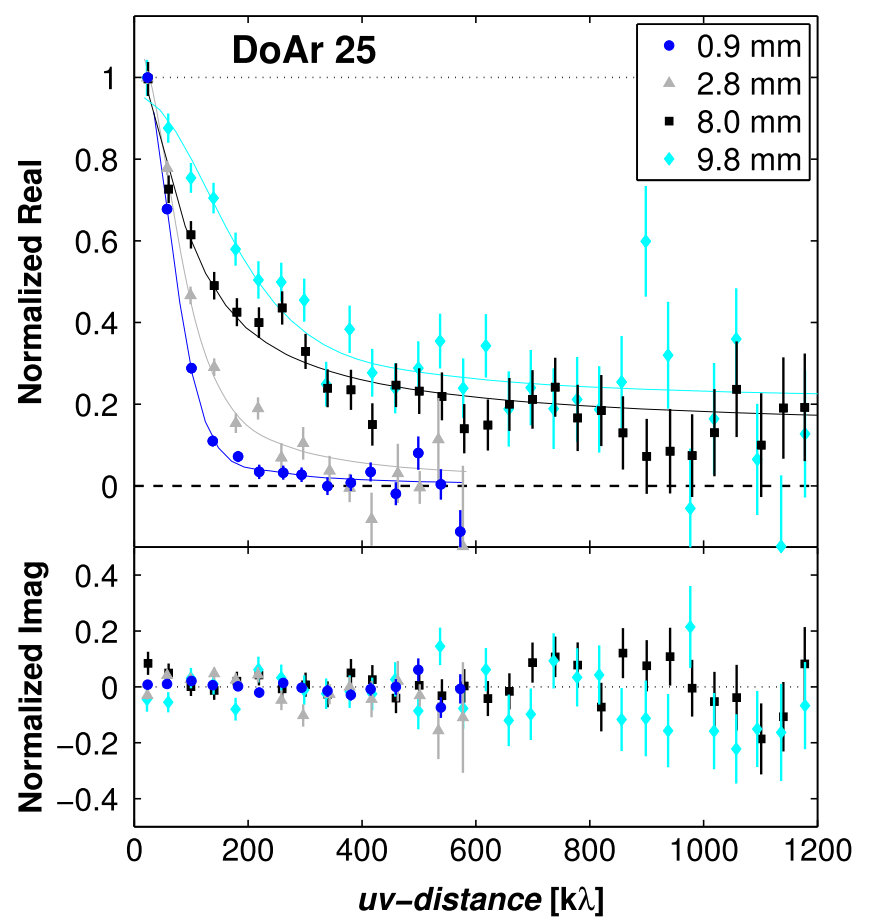

Figure 6. Normalized real and imaginary part of the correlated emission from DoAr 25 as a function of spatial frequency ( $u v$-distance). The visibilities have been de-projected assuming a position angle of $109^{\circ}$ and inclination of $62^{\circ}$, as derived from the modeling of the shortest-wavelength emission. Each bin has a width of $40 \mathrm{k} \lambda$, and has been normalized by the measured flux density at $0-40 \mathrm{k} \lambda$. Filled circles and error bars of different color correspond to the correlated real and imaginary parts of the emission observed at different wavelengths ( $0.9 \mathrm{~mm}$ : blue circle, $2.8 \mathrm{~mm}$ : gray triangle, $8.0 \mathrm{~mm}$ : black square, $9.8 \mathrm{~mm}$ : cyan diamond). The continuous lines correspond to the best-fit disk emission model at each wavelength (see modeling in Section 5.1).

as visibility profiles), for both CY Tau and DoAr 25, at each of the observed wavelengths. Each visibility was deprojected by the known inclination and position angle of the disk (see Section 4 for details of the known disk geometry), before averaging into $u v$-bins with a width of $40 \mathrm{k} \lambda$. To compare the different wavelengths, each visibility profile is normalized by the measured flux density in the first $u v$-bin, between 0 and $40 \mathrm{k} \lambda$. In Figures 5 and 6, the black dotted line at a constant value of $R e=1.0$ represents the observed visibility profile for an unresolved point source. Once a disk is resolved by interferometric observations, its visibility profile will decline from this reference line. Thus, the significant decline of the real part of the visibility profile at each wavelength demonstrates that the emission from both DoAr 25 and CY Tau is resolved, at all the observed wavelengths. Furthermore, a compact source will have a shallower decline with $u v$-distance than an extended source, whose decline will be quite steep. Thus, since the decline of the short-wavelength visibility profile $(0.9,1.3$, and/ or $2.8 \mathrm{~mm}$ ) is steeper than the long-wavelength visibility profile $(7.1,8.0$, and/or $9.8 \mathrm{~mm})$, the disk emission observed at shortwavelengths is more extended than the disk emission observed at long-wavelengths, which has to be more compact. Hence, the differing visibility profiles demonstrate a wavelength-dependent structure in both the CY Tau and DoAr 25 disks, similar to that found for the disks surrounding AS 209 (Pérez et al. 2012), CQ Tau (Banzatti et al. 2011), and several other stars in the Taurus-Auriga star-forming region (Guilloteau et al. 2011). In the next section, we explain the wavelength-dependent 
structure of the emission from CY Tau and DoAr 25 as radial variations of the dust properties across the circumstellar disk.

\section{MODELING OF THE DISK EMISSION}

Observations of CY Tau and DoAr 25 were analyzed using a disk emission model that reproduces the radial brightness distribution at $\mathrm{mm}$ and $\mathrm{cm}$ wavelengths, equivalent to the disk model employed in the analysis of similar observations of the disk surrounding the young star AS 209 (Pérez et al. 2012). At $\lambda=7.1,8.0$, and $9.8 \mathrm{~mm}$ an additional point source at the center of the disk is also included, to represent the potential contamination from sources other than thermal dust emission, as described in Sections 3.1 and 3.2. We adopt the two-layer disk approximation for the disk structure, first presented by Chiang \& Goldreich (1997). In this model, a flared, passively heated disk is irradiated by the central star, with its structure characterized by two components: a disk surface layer that absorbs the stellar radiation, and a disk interior, which is opaque to the stellar photons. Dust in the surface layer will absorb the short-wavelength stellar emission and re-radiate it at longer wavelengths. As the disk interior absorbs the reprocessed emission, the inner regions of the disk can be efficiently heated. We use the implementation of this radiative transfer problem as described by Isella et al. (2009), where the temperature structure of the two-layer model is computed with the iterative method presented by Dullemond et al. (2001). The assumptions implicit in the two-layer disk model break down for the far outer regions of the disk, where the surface density of material drops significantly and even the disk interior becomes optically thin to the stellar radiation. When this condition is met, we assume the same temperature for both the surface layer and disk interior. The disk temperature smoothly decreases as a power-law function of radius down to a minimum of $10 \mathrm{~K}$ in the outer disk, where the dust is heated by the interstellar radiation field.

For the mass surface density structure, $\Sigma(R)$, we adopt the similarity solution for a viscously accreting Keplerian disk subject to the gravity of a massive central object (Lynden-Bell \& Pringle 1974), which can be described by a power law combined with an exponential taper at large radii. If the disk viscosity, $\nu_{v}$, is assumed to be a power-law with radius $\left(\nu_{v} \propto R^{\gamma}\right)$, then the solution for the disk surface density is time-independent (Hartmann et al. 1998). We adopt the same parameterization of the similarity solution presented by Guilloteau et al. (2011):

$$
\Sigma(R)=\Sigma_{0}\left(\frac{R}{R_{0}}\right)^{-\gamma} \times \exp \left(-\left(\frac{R}{R_{C}}\right)^{2-\gamma}\right)
$$

where $\Sigma_{0}$ corresponds to the surface density at radius $R_{0}, \gamma$ is the power-law exponent on the radial dependence of the viscosity, and $R_{C}$ is the characteristic scaling radius. ${ }^{21}$ This prescription for $\Sigma(R)$ behaves as a power law for small radii,

\footnotetext{
21 The scaling radius $R_{C}$ is directly related to the location in the disk where the mass accretion rate changes sign, the transition radius: $R_{t}=R_{C}\left(\frac{1}{2(2-\gamma)}\right)^{1 /(2-\gamma)}$. The transition radius defines a boundary for accretion or expansion, since for $R<R_{t}$ mass flows inward while for $R>R_{t}$ mass flows outward. Note that in the context of viscous disk evolution, the value of the transition radius will increase with time due to conservation of angular momentum: as matter is accreted onto the star the disk must expand to conserve total angular momentum. We include its definition here since different authors will either use $R_{C}$ or $R_{t}$ in their preferred prescription for the disk surface density.
}

while at large radii it decreases smoothly in an exponential fashion.

The inner radius of the disk, $R_{\mathrm{in}}$, is defined as the radius at which the dust sublimates:

$$
R_{\text {in }}=0.07 \mathrm{AU}\left(\frac{L_{\star}}{L_{\odot}}\right)^{1 / 2}\left(\frac{T_{\text {subl }}}{1500 \mathrm{~K}}\right)^{-2}
$$

which corresponds to $\sim 0.04 \mathrm{AU}$ for $\mathrm{CY}$ Tau and $\sim 0.06 \mathrm{AU}$ for DoAr 25, for a dust sublimation temperature of $T_{\text {subl }}=1500 \mathrm{~K}$. Given that the angular resolution of our observations is more than an order of magnitude greater than $R_{\text {in }}$, the value of this parameter does not affect our constraints on the disk structure.

As in the analysis presented by Pérez et al. (2012), we compute the magnitude of the dust opacity, $\kappa_{\lambda}$, using Mie theory. We adopt a grain population of compact spherical grains larger than $a_{\min }=0.01 \mu \mathrm{m}$, in a power-law distribution of grain sizes: $n(a) \propto a^{-q}$ for $a_{\min }<a<a_{\text {max }}$. Grains in the disk interior are assumed to be composed of silicates, organic materials, and water ice, while grains in the disk surface are assumed to be depleted of ice. We obtain optical constants for these grain materials from Semenov et al. (2003; for silicates), Zubko et al. (1996; for amorphous carbon), and Warren (1984; for water ice). Recent studies have revised the solar abundance of oxygen (Asplund et al. 2009), so although we assume the fractional abundances of these materials as given by Pollack et al. (1994), we reallocate the fractions of these astrophysical grains to account for the increased oxygen abundance. This results in grains in the disk interior which are composed of $12 \%$ silicates, $44 \%$ organics, and $44 \%$ water ice, while in the disk surface (where water ice may not be present) the fractional abundances correspond to $21 \%$ silicates and $79 \%$ organics. Except for the normalization of the surface density at radius $R_{0}$ $\left(\Sigma_{0}\right)$, the effect of dust grain composition in the disk modeling will produce a minimal effect in the derived parameters of the disk structure (well within their uncertainties, as shown by Isella et al. 2010). More specifically, although the dust composition does affect the absolute value of opacity, the fact that the dust emission is optically thin at long wavelengths (Section 5.3) means that the derivation of the dust opacity spectral index $\beta$ is insensitive to the assumed absolute opacity value and hence dust composition. This follows since the only frequency dependence when the emission is optically thin corresponds to $S_{\nu} \propto \nu^{\beta} * B_{\nu}(T)$.

Finally, the last parameters that determine the observed disk emission describe the geometry of the disk in the plane of the sky: inclination $(i)$ and position angle (PA, measured from north to east). In the case of CY Tau, the disk geometry has been characterized from molecular line observations of $\mathrm{CO}$ at 0 " 5 resolution: $i=28^{\circ} \pm 5^{\circ}$, PA $=63^{\circ} \pm 1^{\circ}$ (Guilloteau et al. 2011), which is what we adopted for this study (note that these constraints are consistent with those derived from $\mathrm{CN}$ observations at lower spatial resolution: $i=24^{\circ} 0 \pm 2^{\circ} .4$, $\mathrm{PA}=62^{\circ} .5 \pm 1.8$; Guilloteau et al. 2014). For DoAr 25, there are no molecular line observations in the literature. We therefore constrained its disk geometry from our modeling of the dust continuum observations at $0.9 \mathrm{~mm}$, where the signalto-noise ratio is the highest, finding $i=62^{\circ} \pm 1^{\circ}$ and $\mathrm{PA}=109^{\circ} \pm 2^{\circ}$ (consistent with previous modeling by Andrews et al. 2008, 2009).

We find the best-fit model to a single wavelength observation through $\chi^{2}$ minimization using a Markov-Chain 
Monte Carlo (MCMC) procedure (see Isella et al. 2009). The $\chi^{2}$ probability distribution is sampled by varying the free parameters that define the surface density profile $\left(\Sigma_{0}, R_{C}, \gamma\right)$, since the inclination and position angle of the disk are fixed to the values reported above, and where $R_{0}$ has been fixed to a value of $20 \mathrm{AU}$. A set of these parameters defines a model of the disk brightness distribution, with the addition of a point source at the disk center whose flux density corresponds to the estimated level of contamination from non-dust thermal emission, as inferred in Section 3. From this model emission, we produce an image of the disk making sure that the significant spatial scales are covered. We take the Fourier transform of this image and obtain model visibilities sampled at the same locations in the $u v$-domain as the original observations. We compare model and observed visibilities by means of the $\chi^{2}$, and construct 16 separate MCMC chains that sample the parameter space using a Metropolis-Hastings algorithm with a Gibbs sampler.

These chains all converged to the best-fit model, which corresponds to the one that minimizes the $\chi^{2}$ and hence is the model that best reproduces our observations. We note that this MCMC algorithm samples the posterior probability distribution function $(\mathrm{PDF})$ of the parameters when chains have converged and reached equilibrium. Thus, to find best-fit values and confidence intervals for the parameters in our model we adopt a Bayesian approach, marginalizing the resulting PDF over all but one parameter to obtain the probability distribution of the parameter of interest. From each parameter PDF we find the region that contains $68.3 \%(1 \sigma), 95.5 \%(2 \sigma)$, and $99.7 \%(3 \sigma)$ of all samples at equal probability to constrain each of the aforementioned parameters.

\section{MODELING RESULTS}

To model our CY Tau and DoAr 25 observations, we first select the maximum grain size $\left(a_{\max }\right)$ and grain size distribution slope $(q)$ that best reproduces the unresolved SED from sub$\mathrm{mm}$ to $\mathrm{cm}$ wavelengths. For CY Tau, these correspond to $q=3.5$ and $a_{\max }=2.5 \mathrm{~mm}$, which results in an opacity spectral slope $\beta=0.79$ between 1.3 and $7.1 \mathrm{~mm}$. For DoAr 25, the long-wavelength SED can be reproduced with $q=3.5$ and $a_{\max }=1.5 \mathrm{~mm}$, resulting in an opacity spectral slope $\beta=0.91$ between 0.9 and $8.0 \mathrm{~mm}$. Note that while these values of $a_{\max }$ and $\beta$ are consistent with the SEDs, they are relatively poorly constrained by the curvature of the long wavelength emission.

In order to minimize the number of free parameters in this model, we assume a radially constant dust opacity throughout the disk and fit each wavelength separately. Our model then constrains the disk surface density $(\Sigma(R))$ and temperature profile $(T(R))$ at each wavelength; these constraints will appear to be different at different wavelengths if the assumption of a constant dust opacity is not satisfied. The same approach has been successfully employed to measure radial variations of the dust opacity in the disks surrounding RY Tau, DG Tau, and AS 209 (Isella et al. 2010; Pérez et al. 2012); in the following sections we present our results for the circumstellar disks of CY Tau and DoAr 25.

\subsection{CY Tau and DoAr 25 Disk Structure Constraints}

The best-fit and $1 \sigma$ constraints for each model parameter $\left(\Sigma_{0}, R_{C}, \gamma\right)$, obtained from their marginalized PDF, are presented in Tables 5 and 6 . To demonstrate that these best-
Table 5

Best-fit Model Parameters and $1 \sigma$ Constraints for CY Tau

\begin{tabular}{lcccc}
\hline \hline $\begin{array}{l}\lambda \\
(\mathrm{mm})\end{array}$ & $\begin{array}{c}R_{C} \\
(\mathrm{AU})\end{array}$ & $\gamma$ & $\begin{array}{c}\Sigma_{0} \\
\left(\mathrm{~g} \mathrm{~cm}^{-2}\right)\end{array}$ & $\chi_{\text {red }}^{2}$ \\
\hline 1.3 & $62.5_{-1.6}^{+1.8}$ & $0.06_{-0.06}^{+0.06}$ & $2.36_{-0.07}^{+0.07}$ & 1.15 \\
2.8 & $64.4_{-2.1}^{+2.3}$ & $0.16_{-0.07}^{+0.07}$ & $3.32_{-0.12}^{+0.11}$ & 1.14 \\
7.1 & $36.0_{-1.6}^{+2.2}$ & $0.55_{-0.08}^{+0.06}$ & $6.12_{-0.33}^{+0.36}$ & 1.14 \\
\hline
\end{tabular}

Table 6

Best-fit Model Parameters and $1 \sigma$ Constraints for DoAr 25

\begin{tabular}{lcccc}
\hline \hline $\begin{array}{l}\lambda \\
(\mathrm{mm})\end{array}$ & $\begin{array}{c}R_{C} \\
(\mathrm{AU})\end{array}$ & $\gamma$ & $\begin{array}{c}\Sigma_{0} \\
\left(\mathrm{~g} \mathrm{~cm}^{-2}\right)\end{array}$ & $\chi_{\text {red }}^{2}$ \\
\hline 0.9 & $123.2_{-2.8}^{+3.5}$ & $-0.12_{-0.05}^{+0.05}$ & $0.64_{-0.03}^{+0.03}$ & 1.08 \\
2.8 & $103.5_{-4.7}^{+5.9}$ & $0.34_{-0.08}^{+0.07}$ & $1.21_{-0.06}^{+0.05}$ & 1.07 \\
8.0 & $106.0_{-21.7}^{+38.2}$ & $0.83_{-0.13}^{+0.08}$ & $1.28_{-0.08}^{+0.11}$ & 1.11 \\
9.8 & $45.5_{-4.2}^{+7.1}$ & $0.32_{-0.19}^{+0.14}$ & $2.70_{-0.28}^{+0.31}$ & 1.12 \\
\hline
\end{tabular}

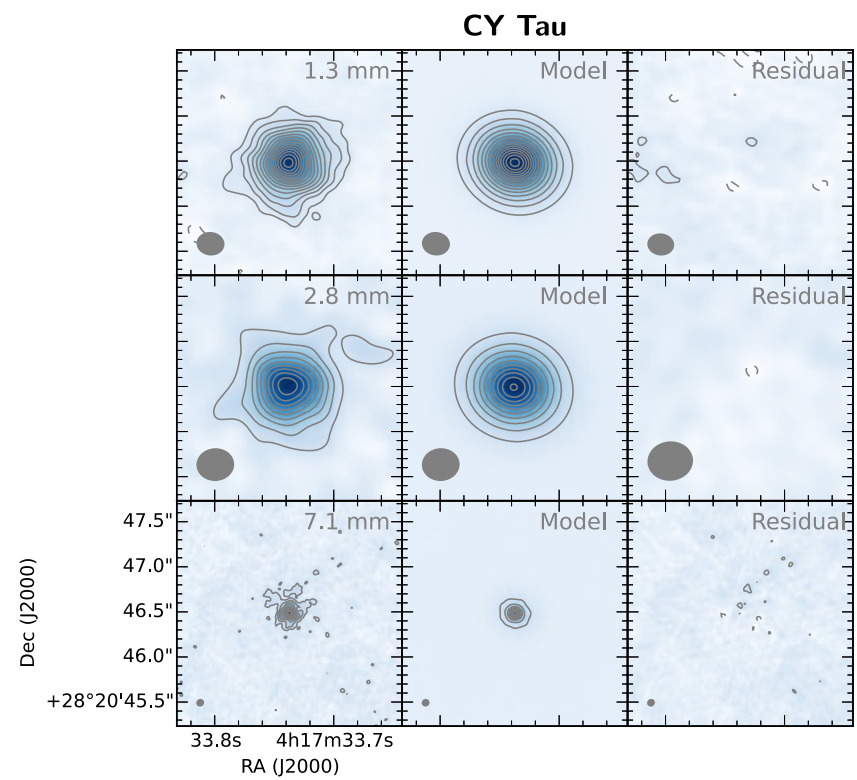

Figure 7. Modeling of the $1.3 \mathrm{~mm}$ (top), $2.8 \mathrm{~mm}$ (middle), and $7.1 \mathrm{~mm}$ (bottom) continuum emission toward CY Tau. For each row, the data are shown on the left, the best-fit model disk is shown on the middle panel, and the residual (obtained by subtracting the best-fit model from the data in the Fourier domain) is on the right. The imaging parameters are as described in Section 3.1. Contours start at $-3 \sigma$, stepping by $3 \sigma$, where $\sigma$ is the rms noise in each map (Table 3).

fit models are sensible representations of the observations, we imaged the model and residual visibilities (calculated by subtracting the model visibilites from the data) in the same way as the original observations. The resulting images are shown in Figures 7 and 8 for CY Tau and DoAr 25 respectively. Additionally, for each of the best-fit models, we computed de-projected visibility profiles (solid lines in Figures 5 and 6). The adopted model is a good representation of the data at each separate wavelength, as no significant residual emission can be seen in the residual maps and the visibility profile for our bestfit model agrees with the observations at all wavelengths.

From each separate model fitting we characterize the disk surface density and temperature profile $(\Sigma(R)$ and $T(R))$ at each wavelength. Figures 9 and 10 present the best-fit and $3 \sigma$ constraints on the disk temperature, surface density, and optical 


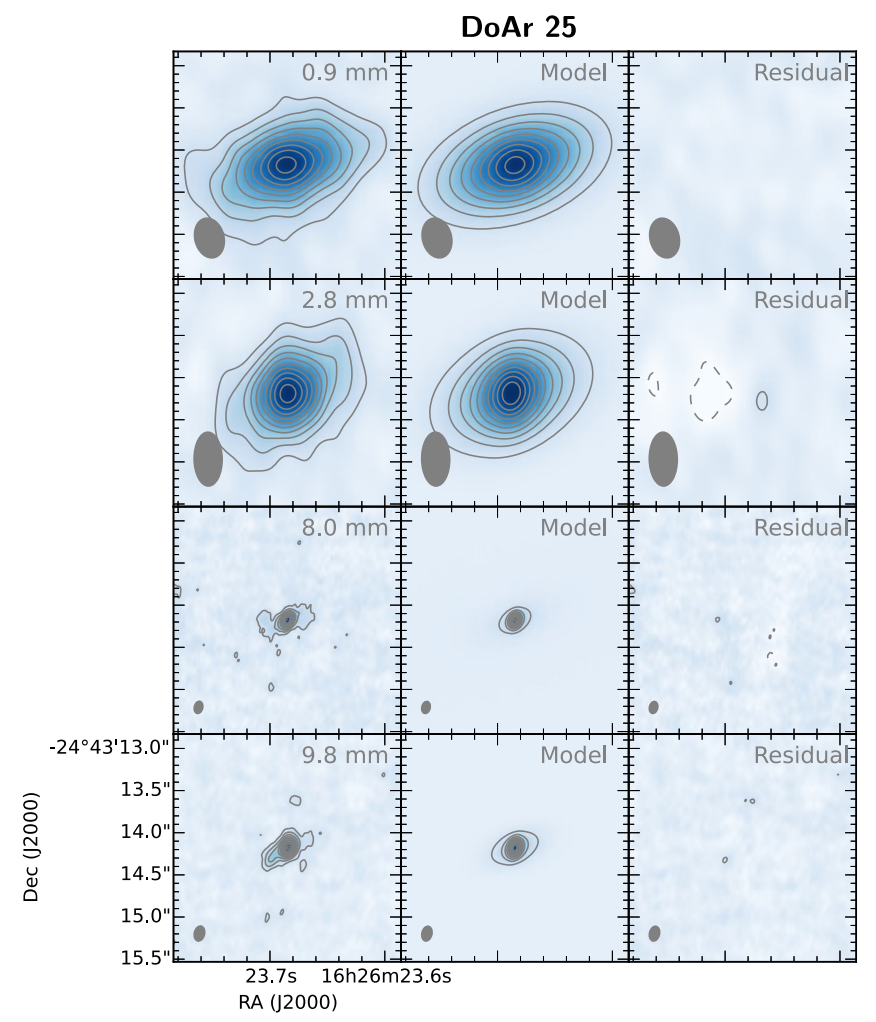

Figure 8. Modeling of the $0.9 \mathrm{~mm}$ (top), $2.8 \mathrm{~mm}$ (2nd from top), 8.0 and $9.8 \mathrm{~mm}$ (bottom two rows) continuum emission toward DoAr 25. For each row, the data are shown on the left, the best-fit model disk is shown on the middle panel, and the residual (obtained by subtracting the best-fit model from the data in the Fourier domain) is on the right. The imaging parameters are as described in Section 3.2. Contours start at $-3 \sigma$, stepping by $3 \sigma$, where $\sigma$ is the rms noise in each map (Table 4).

depth $\left(\tau_{\lambda}=\kappa_{\lambda} \Sigma(R)\right)$, obtained from modeling each wavelength independently. The temperature profiles inferred from observations at different wavelengths are very similar (this is expected, since dust of different sizes in the disk should have reached radiative equilibrium and be at the same temperature). In particular, the best-fit temperature at each wavelength differs from the mean temperature profile by at most $\sim 3 \mathrm{~K}$ for $\mathrm{CY}$ Tau and $\sim 5 \mathrm{~K}$ for DoAr 25 . However, the surface density profiles that were inferred separately from each observation (middle panels of Figures 9 and 10), are different at each observed wavelength. This is clearly not physical (the dust emission arises from the same disk at all wavelengths!), and cannot be reconciled through a global change in the dust opacity. We conclude that we need to consider a change in the dust opacity as a function of location (specifically, radius) in the disk, in order to explain the observed dust emission. This is explored further in Section 5.2 below. Finally, the right panels of Figures 9 and 10 show the inferred optical depth, demonstrating that the dust emission is optically thin at all wavelengths for $R>15$ AU (well within our spatial resolution at all wavelengths for both disks). Furthermore, the emission would be optically thin even if the mid-plane dust temperature were lower by a factor of a few.

\subsection{Radial Variations of the Dust Opacity}

Since the dust emission is in the optically thin regime for both CY Tau and DoAr 25 (right panel of Figures 9 and 10), the observed emission will directly depend on three physical quantities: the dust opacity, the dust mass surface density, and the temperature, such that $S_{\nu} \propto \kappa_{\lambda} \Sigma B_{\nu}(T)$. Thus, our observations provide a constraint for the product $\kappa_{\lambda} \times \Sigma \times B_{\nu}(T)$, where the opacity, surface density, and temperature may vary with radius, but only the dust opacity is a function of the observed wavelength. Our model fitting presented abovewhich assumes a constant dust opacity with radius and where each wavelength is fitted separately-results in a wavelengthdependent $\Sigma(R)$, signifying that the assumption of a radially constant dust opacity is not warranted by the data. To reconcile these wavelength-dependent $\Sigma_{\lambda}(R)$ and $T_{\lambda}(R)$ (Figures 9 and $10)$, as well as the wavelength-dependent disk structure seen in the normalized visibility profiles (Figures 5 and 6) we require a change in the dust properties as a function of radial distance from the central star, for both the CY Tau and DoAr 25 disks.

We focus on the interpretation that the spectral index of the dust opacity, $\beta$, is changing with radius, which we obtain following the procedure described by Pérez et al. (2012) and Isella et al. (2010), and summarize here. Since the constraints found in Section 5.1 reproduce the data well, these must be consistent with the true (but unknown) disk structure:

$$
\kappa_{\lambda} \Sigma_{\lambda}(R) B_{\nu}\left(T_{\lambda}(R)\right)=\bar{\kappa}_{\lambda}(R) \bar{\Sigma}(R) B_{\nu}(\bar{T}(R))
$$

where $\bar{\kappa}_{\lambda}(R), \bar{\Sigma}(R)$, and $\bar{T}(R)$ are the true disk physical quantities, and the left-hand side of this equation corresponds to our multi-wavelength constraints for the disk structure. Thus, two different wavelengths, $\lambda_{1}$ and $\lambda_{2}$ (and corresponding frequencies, $\nu_{1}$ and $\nu_{2}$ ), can be related by

$$
\left(\frac{\lambda_{1}}{\lambda_{2}}\right)^{-\beta(R)}=\left(\frac{\lambda_{1}}{\lambda_{2}}\right)^{-\beta_{C}} \times \frac{\Sigma_{\lambda_{1}}(R) \frac{B_{\nu_{1}}\left(T_{\lambda_{1}}(R)\right)}{B_{\nu_{1}}(\bar{T}(R))}}{\Sigma_{\lambda_{2}}(R) \frac{B_{\nu_{2}}\left(T_{\lambda_{2}}(R)\right)}{B_{\nu_{2}}(\bar{T}(R))}}
$$

assuming that at long wavelengths the dust opacity follows a power-law behavior $\left(\kappa_{\lambda} \propto \lambda^{-\beta}\right)$, and $\beta_{C}$ corresponds to the spectral slope of the assumed constant dust opacity with radius, derived from the integrated SED used in the initial models. A useful prescription to infer radial variations of the opacity spectral slope $\left(\Delta \beta(R)=\beta(R)-\beta_{C}\right)$ in dual-wavelength observations is derived from this last equation (see also Equation (9) from Isella et al. 2010):

$$
\Delta \beta(R)=-\log ^{-1}\left[\frac{\lambda_{1}}{\lambda_{2}}\right] \times \log \left[\frac{\Sigma_{\lambda_{1}} B_{\nu_{1}}\left(T_{\lambda_{1}}\right) / B_{\nu_{1}}(\bar{T})}{\Sigma_{\lambda_{2}} B_{\nu_{2}}\left(T_{\lambda_{2}}\right) / B_{\nu_{2}}(\bar{T})}\right]
$$

where the dust surface density and temperature depend on the distance from the star $R$. Equation (5) implies that in logarithmic space $\Delta \beta(R)$ is the slope of a straight line at points $\left\{x=\log (\lambda), y=\log \left(\Sigma_{\lambda} B_{\nu}\left(T_{\lambda}\right)\right) / B_{\nu}(\bar{T})\right\}$.

Note that if the emission is in the R-J domain: $B_{\nu}(T) \propto T$, and thus the true dust temperature $(\bar{T}(R))$ will not be needed to derive $\Delta \beta(R)$. However, at cold temperatures in the outer disk and/or at short wavelengths, the R-J assumption breaks down and we require an estimate of $\bar{T}(R)$. As mentioned in Section 5.1, the temperature constraints from these multiwavelength data are quite similar, thus we assume the true dust temperature to be the mean $T_{\lambda}(R)$, shown as a dashed line on the left panels of Figures 9 and 10. Note that a change in the estimate of $\bar{T}(R)$ will have a minimal impact in the $\Delta \beta(R)$ 

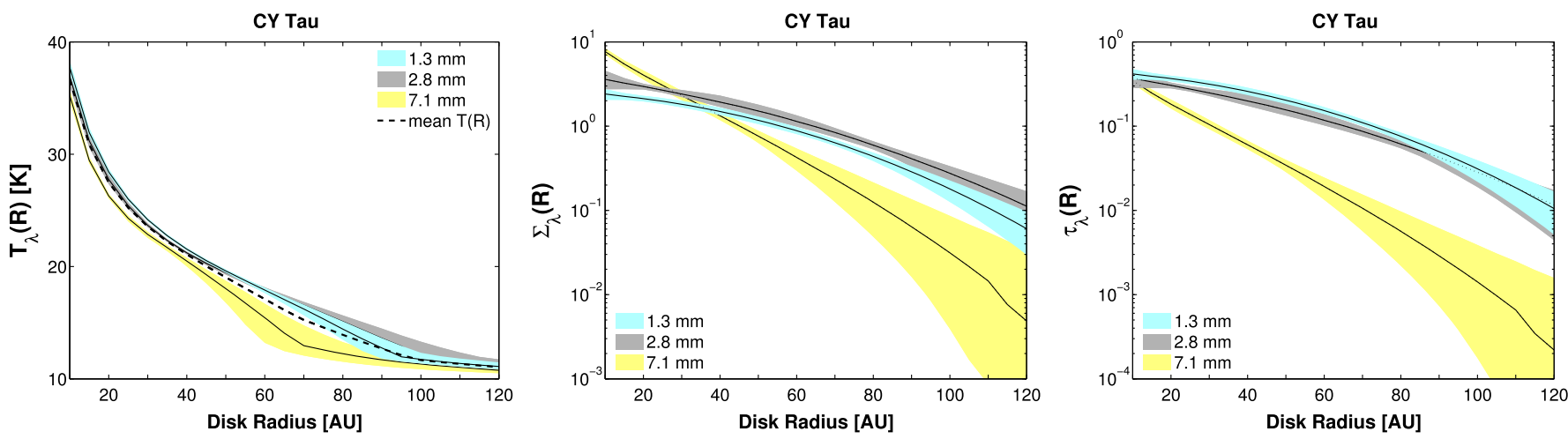

Figure 9. Left and middle: $T_{\lambda}(R)$ and $\Sigma_{\lambda}(R)$ inferred from separate modeling of our multi-wavelength observations of CY Tau, assuming a constant dust opacity with radius. Right: optical depth $\tau_{\lambda}(R)=\kappa_{\lambda} \times \Sigma_{\lambda}(R)$ inferred from separate modeling of CY Tau multi-wavelength observations, assuming a radially constant $\kappa_{\lambda}$. Colored regions: $3 \sigma$ confidence interval constrained by our observations; continuous line: best-fit model; dashed line on left panel: mean temperature profile. The different $\Sigma(R)$ and $T(R)$ profiles for each wavelength imply a varying dust opacity with radius, and because of this, none of them is the true surface density and temperature profile of the disk.
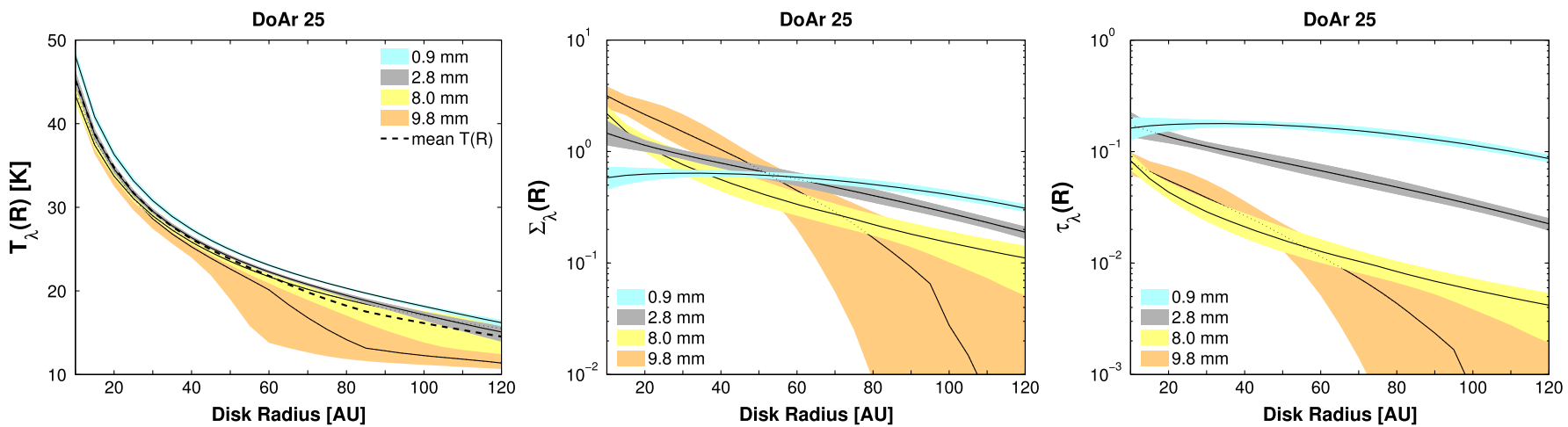

Figure 10. Left and middle: $T_{\lambda}(R)$ and $\Sigma_{\lambda}(R)$ inferred from separate modeling of our multi-wavelength observations of DoAr 25, assuming a constant dust opacity with radius. Right: optical depth $\tau_{\lambda}(R)=\kappa_{\lambda} \times \Sigma_{\lambda}(R)$ inferred from separate modeling of DoAr 25 multi-wavelength observations, assuming a radially constant $\kappa_{\lambda}$. Colored regions: $3 \sigma$ confidence interval constrained by our observation; continuous line: best-fit model; dashed line on left panel: mean temperature profile. The different $\Sigma(R)$ and $T(R)$ profiles for each wavelength imply a varying dust opacity with radius, and because of this, none of them is the true surface density and temperature profile of the disk.

constraint, as $\bar{T}(R)$ only appears in the ratio of Planck functions evaluated at two different wavelengths in Equation (5) (and close to the $\mathrm{R}-\mathrm{J}$ limit the dependence with $\bar{T}(R)$ is negligible). Nevertheless, we tested two different assumptions where $\bar{T}(R)$ was equal to twice and half the mean of $T_{\lambda}(R)$. These substantial changes of $\times 2$ only changed our constraints in $\Delta \beta(R)$ by $\sim 10 \%$ or less throughout the disk.

Since the result of the MCMC algorithm is a sampled posterior PDF for the parameter space, we can construct a PDF for the product $\Sigma_{\lambda} \times B_{\nu}\left(T_{\lambda}\right) / B_{\nu}(\bar{T})$ at each radius and for each wavelength that has been modeled. To determine $\Delta \beta(R)$ we use a Monte Carlo simulation, where the PDF of $\Sigma_{\lambda} \times B_{\nu}\left(T_{\lambda}\right) / B_{\nu}(\bar{T})$ is randomly sampled at each wavelength; a random sampling of $\Delta \beta(R)$ will thus correspond to the slope of the line that intersects the points $\{x=\log (\lambda)$, $\left.y=\log \left(\Sigma_{\lambda} B_{\nu}\left(T_{\lambda}\right)\right) / B_{\nu}(\bar{T})\right\}$. By obtaining a large number of random samples of $\Sigma_{\lambda} \times B_{\nu}\left(T_{\lambda}\right) / B_{\nu}(\bar{T})$ we populate the PDF of $\Delta \beta(R)$ at each radius. We find the best-fit value of $\Delta \beta$ at a radius $R$ from the peak of this PDF, and we derive confidence intervals from the region of the distribution that contains $68.3 \%, 95.5 \%$, and $99.7 \%$ of all samples at equal probability (as described in Section 4 for the uncertainties of the model parameters). These steps are performed for all radii sampled by the data, in order to obtain a best-fit value and inferred constraints on $\beta(R)=\beta_{C}+\Delta \beta(R)$ for CY Tau and DoAr 25 .
Figures 11 and 12 present the observational constraint on $\beta$ as a function of radius, obtained for the circumstellar disks of CY Tau and DoAr 25. The values of $\beta$ allowed by these multiwavelength observations are significantly different from (and below) the ISM value of the dust opacity slope $\left(\beta_{\text {ISM }} \sim 1.7\right)$ for $R \lesssim 60 \mathrm{AU}$ in the case of CY Tau, and $R \lesssim 70$ AU for DoAr 25 . Furthermore, a gradient on $\beta$ with radius is found in both disks. This gradient can only be consistent with a constant value of $\beta$ when the uncertainties in $\beta(R)$ are extended to $7 \sigma$. We note that the uncertainty in the absolute flux density scale will potentially introduce an additional systematic offset in the constrained values of $\beta(R)$. But because a fractional uncertainty in the flux density scale affects all radii equally at a particular wavelength, the overall shape and significance of the deviation in $\beta(R)$ from a constant value are not affected by uncertainties in the flux density scale. Given the 10\%-15\% uncertainty in the absolute flux density scales for the three telescopes used, we estimate that the level of the systematic offset introduced to be $\sim 0.16$ for CY Tau and $\sim 0.08$ for DoAr 25, indicated by a vertical errorbar in the bottom-right corner of Figures 11 and 12. This systematic uncertainty is smallest for DoAr 25, because observations of this disk were obtained at wavelengths that are further separated than for CY Tau, reducing the uncertainty due to the increase in the lever arm used to infer $\beta$. 


\section{CY Tau}

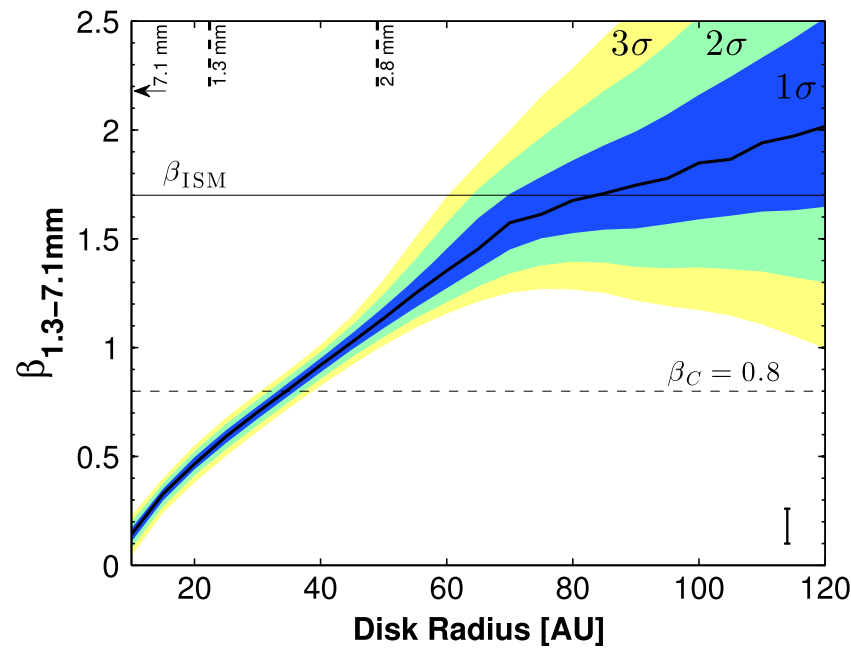

Figure 11. Spectral index of the dust opacity $(\beta)$ as a function of radius, inferred from multi-wavelength observations of the CY Tau disk. Black line: best-fit $\beta(R)$; colored areas: confidence interval constrained by our observations. The vertical dashed-lines represent the smallest spatial scale probed at each wavelength, the arrow indicates that at $7.1 \mathrm{~mm}$ the smallest spatial scale is outside of the range plotted in this figure, corresponding to 42 mas or $6 \mathrm{AU}$. The errorbar at the bottom right indicates the systematic uncertainty on $\beta(R)$ resulting from absolute flux scale uncertainty.

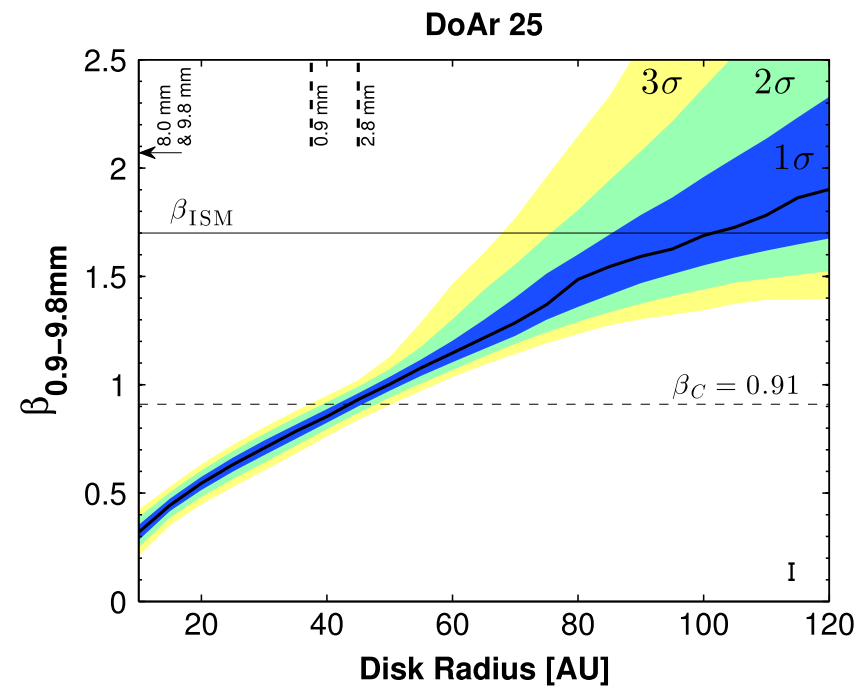

Figure 12. Spectral index of the dust opacity $(\beta)$ as a function of radius, inferred from multi-wavelength observations of the DoAr 25 disk. Black line: best-fit $\beta(R)$; colored areas: confidence interval constrained by our observations. The vertical dashed-lines represent the smallest spatial scale probed at each wavelength, the arrow indicates that at 8.0 and $9.8 \mathrm{~mm}$ the smallest spatial scale is outside of the range plotted in this figure, corresponding to 48 mas or $6 \mathrm{AU}$ at $8.0 \mathrm{~mm}$, and $58 \mathrm{mas}$ or $7 \mathrm{AU}$ at $9.8 \mathrm{~mm}$. The errorbar at the bottom right indicates the systematic uncertainty on $\beta(R)$ resulting from absolute flux scale uncertainty.

\subsection{Radial Variation of the Dust Opacity under the Assumption of Grain Growth}

As discussed in the introduction, the inferred changes in the dust opacity for CY Tau and DoAr 25 may arise from changes in grain size, composition, or grain geometry. Draine (2006) explored the frequency dependence of the dust opacity for different materials which may have enhanced emissivity at long wavelengths and thus can create low values of $\beta$ with small grains. He concluded that changes in composition are unlikely to explain $\beta<1$ and advocates for changes in the grain-size distribution to explain the common finding of $\beta \lesssim 1$ in unresolved observations of protoplanetary disks (for references see Section 1). On the other hand, changes in grain porosity can produce low values of $\beta$ (see, e.g., Figure 11 of Kataoka et al. 2014). However, the low filling factor needed to reach $\beta<1$ requires quite large fluffy grains (e.g., larger than meter-size for filling factors of $10^{-1}-10^{-2}$ ). Given the complexity of exploring changes in composition, geometry, and size of dust grains simultaneously, and given that the option of large grains can more simply reproduce the observed changes in $\kappa_{\lambda}$, we assume that the radial variations of the dust opacity (observed as changes in $\beta$ in the previous section) are only caused by changes in $a_{\max }$ with radius. We now estimate the range of allowed maximum grain sizes at each location of the disk for CY Tau and DoAr 25.

As discussed by Pérez et al. (2012), the determination of $\beta(R)$ depends on the assumption that the dust opacity follows a power law with wavelength: $\kappa_{\lambda} \propto \lambda^{-\beta}$. This essential assumption might not be the case, as non-power-law dependence with wavelength in $\kappa_{\lambda}$ can be seen on dust populations which are limited to a maximum grain size $\left(a_{\max }\right)$ of sub-millimeter and $\mathrm{mm}$-sized particles (see, e.g., Draine 2006). Thus, variations in the dust properties of these disks, particularly $a_{\max }$, should be directly constrained without this assumption.

Following the method outlined by Pérez et al. (2012), we find the best-fit dust opacity (which depends on $a_{\max }$ ), along with the best-fit surface density, that is needed to reproduce the constraints on $\kappa_{\lambda} \times \Sigma_{\lambda} \times B_{\nu}\left(T_{\lambda}\right)$ found in Section 5.1. Since the temperature profile at each wavelength $\left(T_{\lambda}\right)$ is found to be similar at all wavelengths, we assume the dust temperature is equal to the mean $T_{\lambda}$ at each radius (see dashed line on left panel of Figures 9 and 10). At this point, we include the uncertainty in the absolute flux density scale in our modeling, which ranges between $10 \%$ and $15 \%$ for the three telescopes used. This improvement to our method is important, as it introduces another source of error in the constraints obtained from the flux density at each wavelength, and impacts the error budget in the characterization of radial variations of the maximum grain size. We note that the uncertainty in the flux density scale as a function of wavelength was not included in our previous analysis of AS 209 (e.g., Pérez et al. 2012). For each MCMC realization that reproduces the disk surface brightness as a function of radius, we draw a random number from a normal distribution centered at 1.0 and whose standard deviation $(\sigma)$ corresponds to the flux scale uncertainty. Depending on the wavelength, this would correspond to $\sigma=0.1$ or $\sigma=0.15$. We then scale the surface density normalization of each MCMC realization by a different random number, ensuring that the uncertainty in the absolute flux density scale is reflected in the resulting probability distribution.

Assuming the same dust composition as in Section 4 we place $3 \sigma$ confidence limits for $a_{\max }(R)$ and $\bar{\Sigma}(R)$, while exploring two representative grain size distributions: a steep distribution with $q=3.5$ and a shallow distribution with $q=2.5$. The chosen distributions are motivated by the resulting size-distribution of a collisional cascade (Dohnanyi 1969) and the measured grain size distribution in the ISM (Mathis et al. 1977), which are both characterized by $n(a) \propto a^{-3.5}$, while a less steep distribution of $q=2.5$ is selected to observe the effects of a reduced total number of smaller particles. These 

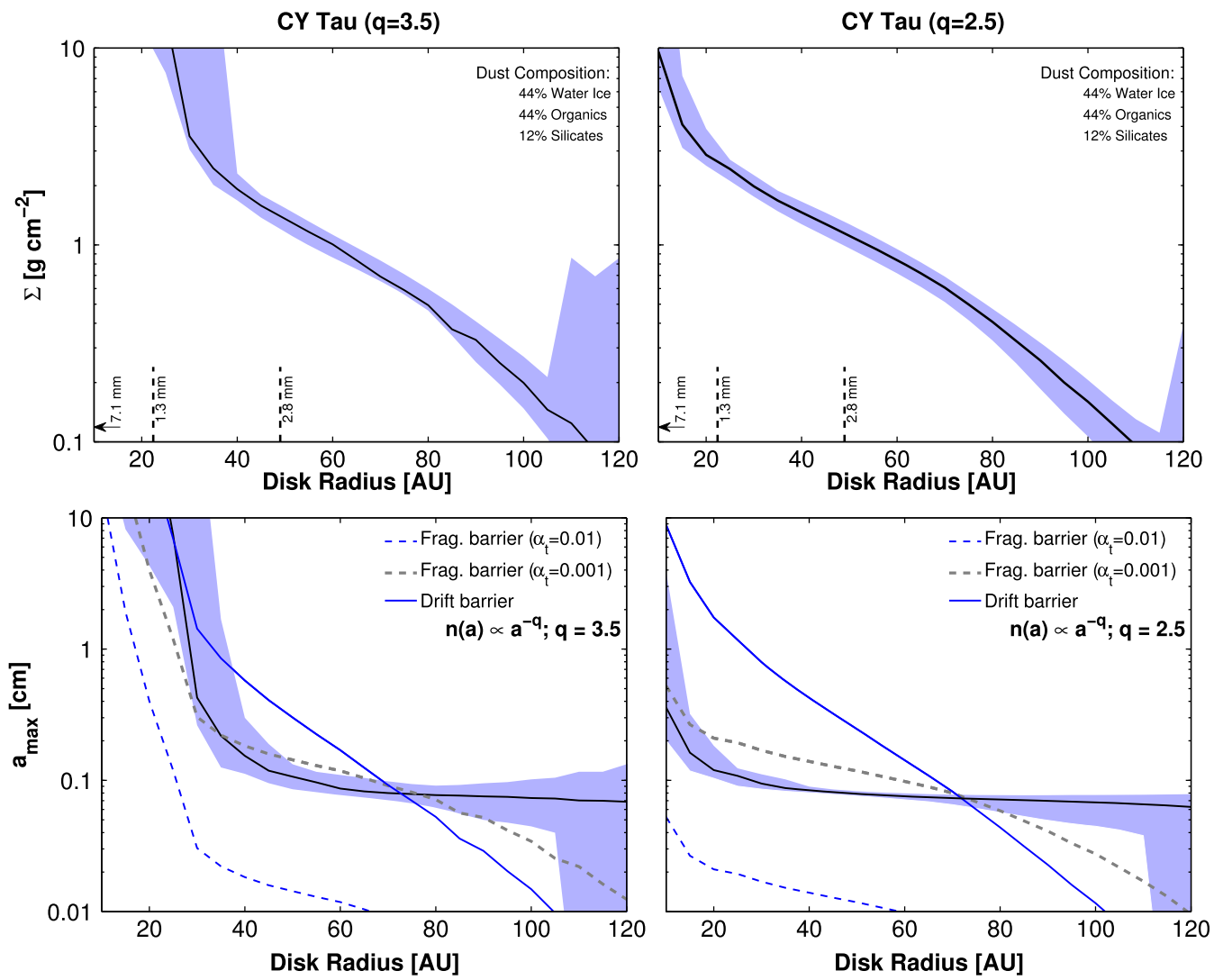

Figure 13. Constraints for the CY Tau disk on the surface density (top panels) and maximum grain size (bottom panels), for two grain size distributions $q=3.5$ (left panels), $q=2.5$ (right panels). The best-fit $\Sigma(R)$ (top) and $a_{\max }(R)$ (bottom) are indicated by a continuous black line, while the shaded region represents our confidence interval at $3 \sigma$. We compare our observational constraints with theoretical grain evolution models by Birnstiel et al. (2012) that include fragmentation (dashed lines, bottom panels), or radial drift (blue continuous line, bottom panels). For the fragmentation barrier we explore two different levels of turbulence in the CY Tau disk: $\alpha_{t}=0.01$ (blue dashed line) and $\alpha_{t}=0.001$ (gray dashed line), with $v_{\text {frag }}=10 \mathrm{~m} \mathrm{~s}^{-1}$ in both cases. The smallest spatial resolution probed at each wavelength is indicated by a vertical dashed line in the top panels (at $7.1 \mathrm{~mm}$, the smallest spatial scale corresponds to 42 mas or 6 AU, outside of the range plotted in this figure and indicated by an arrow).

results are presented as the shaded regions in Figures 13 and 14 for CY Tau and DoAr 25, respectively. We find that within $\sim 100$ AU from the central protostar, grains have grown up to at least up to $\sim 0.4 \mathrm{~mm}$ for CY Tau and $\sim 0.2 \mathrm{~mm}$ for DoAr 25 . The best-fit $a_{\max }(R)$ displays a gradient with radius, where smaller grains are present in the outer disk and larger grains are present in the inner disk. However, the rise of $a_{\max }$ toward smaller radii strongly depends on the assumed slope of the grain size distribution. This occurs because a shallow grain-size distribution will have more large grains (by number) than a steep distribution. Since large grains dominate the emission at these wavelengths, we can reproduce the observed emission with a maximum grain size that can be smaller for a shallow distribution than for a steep distribution. This explains the difference in our $a_{\max }$ constraints for the representative slopes $q=2.5$ and $q=3.5$ that we selected. Unfortunately, current observations do not have the necessary sensitivity to discern the value of the grain size distribution slope.

\section{DISCUSSION}

\subsection{Comparison with Theory}

Birnstiel et al. (2012) presented simple analytical forms for the variation of $a_{\max }$ with radius for two different regimes: when a population of dust grains is limited by fragmentation of dust particles, whose differential velocity is driven by turbulence in the disk:

$$
a_{\max }^{\mathrm{frag}}(R) \sim \frac{2}{3 \pi} \frac{\sum_{\mathrm{gas}}(R)}{\rho \alpha_{t}} \frac{v_{\mathrm{frag}}^{2}}{c_{s}^{2}(R)}
$$

and when a population of dust grains is limited by radial drift, as larger particles are removed from the population and accreted onto the star due to gas drag:

$$
a_{\max }^{\mathrm{drift}}(R) \sim \frac{2}{\pi} \frac{\Sigma_{\mathrm{dust}}(R)}{\rho} \frac{v_{\mathrm{K}}^{2}(R)}{c_{s}^{2}(R)}\left|\frac{d \ln P}{d \ln R}\right|^{-1} .
$$

Here $\Sigma_{\text {gas }}$ and $\Sigma_{\text {dust }}$ are the gas and dust surface density profile, $\rho$ is the dust grain internal density, $\alpha_{t}$ is the disk turbulence parameter as presented by Shakura \& Sunyaev (1973), $v_{\text {frag }}$ is the velocity at which a collision between dust grains will result in fragmentation rather than growth, $v_{\mathrm{K}}$ is the local Keplerian velocity, and $c_{s}$ is the local sound speed of the gas.

Employing our constraints on the disk surface density, which assume a constant gas-to-dust ratio of 100:1 over the entire disk, we compared our derived observational constraints on $a_{\max }$ versus radius with these grain growth models, adopting standard values for the turbulence $\left(\alpha_{t}=0.01\right)$, dust density ( $\rho=1.3 \mathrm{~g} \mathrm{~cm}^{-3}$, from the Pollack et al. (1994) abundances adopted in this paper), and fragmentation velocity $\left(v_{\text {frag }}=10 \mathrm{~m} \mathrm{~s}^{-1}\right.$ Birnstiel et al. 2012). Also, we verify that $\mathrm{mm}$-sized particles are in the Epstein regime throughout the 

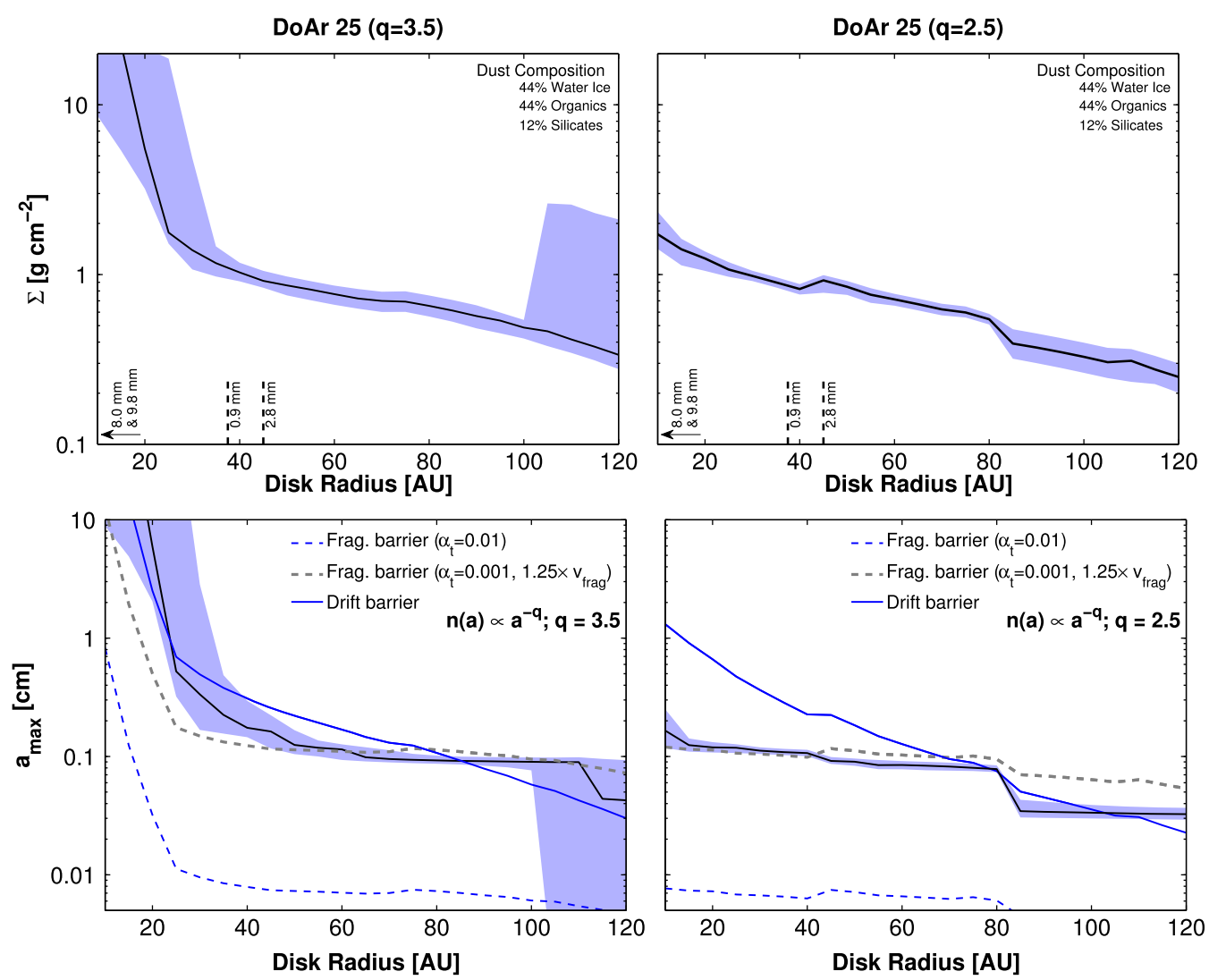

Figure 14. Constraints for the DoAr 25 disk on the surface density (top panels) and maximum grain size (bottom panels), for two grain size distributions $q=3.5$ (left panels), $q=2.5$ (right panels). The best-fit $\Sigma(R)$ (top) and $a_{\max }(R)$ (bottom) are indicated by a continuous black line, while the shaded region represents our confidence interval at $3 \sigma$. We compare our observational constraints with theoretical grain evolution models by Birnstiel et al. (2012) that include fragmentation (dashed lines, bottom panels), or drift (blue continuous line, bottom panels). For the fragmentation barrier we explore two different levels of turbulence and fragmentation velocity in the DoAr 25 disk: $\alpha_{t}=0.01$ with $v_{\text {frag }}=10 \mathrm{~m} \mathrm{~s}^{-1}$ (blue dashed line), and $\alpha_{t}=0.001$ with $v_{\text {frag }}=12.5 \mathrm{~m} \mathrm{~s}^{-1}$ (gray dashed line). The spatial resolution at each wavelength is indicated by a vertical dashed line in the top panels (FWHM for 8.0 and $9.8 \mathrm{~mm}$, HWHM for 2.8 and $0.9 \mathrm{~mm}$ ).

disk (with a Stokes number $\mathrm{St}<1$ ), which is a necessary condition for the use of Equation (7) as the size limit in radial drift. For this particular set of turbulence and fragmentation parameters, the theoretical $a_{\max }$ curves for CY Tau and DoAr 25 are shown in Figures 13 and 14, respectively, where the continuous line illustrates the maximum grain size for a drift-dominated population, and the dashed blue line correspond to a fragmentation-dominated population. Note that since our constraint on the surface density with radius depends on the adopted grain size distribution slope, $q$, the drift and fragmentation barriers will depend on $q$ as well.

In both disks and with this standard set of parameters, the fragmentation barrier under-predicts the maximum grain size achievable. Following Equation (6), a better correspondence could be found if: (1) the disk turbulence were lowered to $\alpha_{t} \sim 10^{-3}-10^{-4}$, or (2) the gas-to-dust ratio were higher than 100:1 by an order of magnitude, or (3) the critical velocity for fragmentation were higher than $v_{\text {frag }}=10 \mathrm{~m} \mathrm{~s}^{-1}$. This last option seems unlikely if grains are compact, given that threshold velocities for fragmentation and/or bouncing of up to a few $\mathrm{m} \mathrm{s}^{-1}$ have been measured experimentally for compact centimeter and decimeter-sized grains (see, e.g., Deckers \& Teiser (2013) for silicate grains, Heißelmann et al. (2010) and Hill et al. (2015) for icy grains, and Blum \& Wurm (2008) for a review). On the other hand, if grains are porous (e.g., Kataoka et al. 2014), fragmentation could occur at higher threshold velocities. However, porous grains have larger cross sections resulting in mass loss through collisional erosion rather than fragmentation, which similarly halts the growth of these grains (Krijt et al. 2015). Now, the second option of a higher gas-todust ratio needs to be pursued with measurements of the total gas mass in these disks in particular, but this alternative also seems unlikely given the recent results in the Taurus-Auriga star-forming region where low gas-to-dust ratios (well below the ISM standard of 100:1) are inferred for a large number of circumstellar disks by Williams \& Best (2014). Finally, the first option of a quiescent disk of low turbulence $\left(\alpha_{t}<0.01\right)$ for the disks around CY Tauand DoAr 25(and also AS 209) seems plausible, and would further augment grain growth. We test this option by calculating the expected maximum grain size when the disk turbulence is an order of magnitude lower $\left(\alpha_{t}=0.001\right)$, and we find a better correspondence with our $a_{\text {max }}$ constraints on CY Tau and DoAr 25 (for this last disk we also increased the fragmentation velocity by $25 \%$ ). Note that values of $\alpha_{t} \sim 10^{-3}-10^{-4}$ would still produce turbulent relative velocities high enough for fragmentation, a condition attained when $\alpha_{t}>\frac{1}{3}\left(\frac{v_{\text {frag }}}{c_{s}}\right)^{2}$, and confirmed for both CY Tau and DoAr 25. The prediction of low turbulence should be tested with future observations of the turbulent linewidth in these circumstellar disks.

At the same time, the radial drift barrier roughly agrees with our $a_{\max }$ inference in both disks. For DoAr 25, the growth barrier imposed by a radial drift of macroscopic particles is within a factor of a few of the observational constraint, and the 

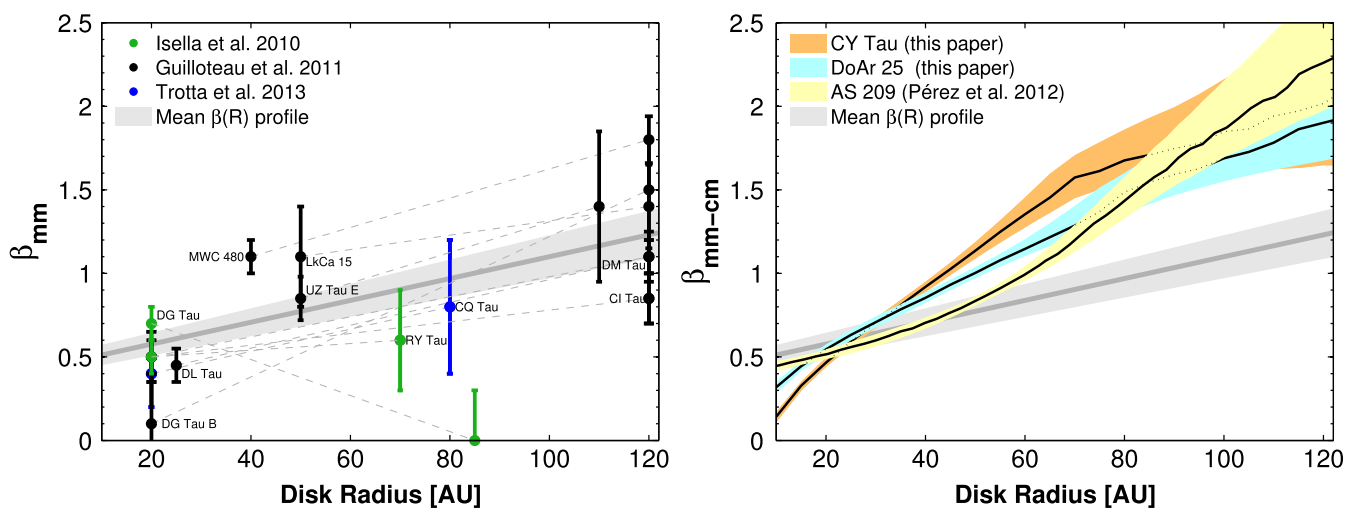

Figure 15. Left: observational constraints on the spectral index of the dust opacity $\beta$, with $1 \sigma$ error bars, as measured from published resolved millimeter observations, where all but CQ Tau have been observed only at 1 and $3 \mathrm{~mm}$ (Isella et al. 2010; Guilloteau et al. 2011; Trotta et al. 2013). Note that the majority of the previously available constraints are still consistent with a constant value of $\beta$ across the disk at $3 \sigma$. The mean profile of $\beta(R)$ based on these observations is shown by the gray shaded region. Right: the radial variation of $\beta$ for the three circumstellar disks analyzed in a consistent fashion: CY Tau and DoAr 25 (this paper), and AS 209 (Pérez et al. 2012), where the shaded regions show the $1 \sigma$ constraints. We note that at the $3 \sigma$ level the 3 disks analyzed here at consistent with each other and with the average $\beta(R)$ profile from the literature.

same occurs for CY Tau at least up to 80 AU. Most likely it is both mechanisms that are at play simultaneously in these disks. Finally, we note that at a radii $\gtrsim 100 \mathrm{AU}$, the population of grains in CY Tau is above both barriers by a factor of few at least, and this is the case for both a shallow or a steep grain size distributions. This result is readily appreciated in the CY Tau data, where millimeter-wave emission at 1.3 and $2.8 \mathrm{~mm}$ is observed at large distances from the star, indicating the presence of mm-sized grains at $\sim 100 \mathrm{AU}$. If the gas surface density distribution were different from the dust surface density we measured, the pressure gradient term $\left|\frac{d \ln P}{d \ln R}\right|$ in Equation (7) would change, and thus a region of higher gas pressure, i.e., a local maximum in $P(R)$, could trap mm-sized dust grains in the outer disk of CY Tau. In such a scenario, grains can overcome the radial drift barrier that is expected when the gas distribution just monotonically increases with radius (e.g., Pinilla et al. 2012b). The origin of these pressure enhancements is strongly debated: locally they may arise from turbulent eddies (Klahr \& Henning 1997; Johansen \& Klahr 2005), while on a global disk scale they may emerge from Rossby-wave instabilities (Lovelace et al. 1999; Li et al. 2000), long-lived vortices (Barge \& Sommeria 1995), or from the gap opened by one or multiple planets (Pinilla et al. 2012a). However, higher angular resolution observations of the distribution of small and large grains, as well as observations of tracers in the gas emission, are needed to understand what is the likely cause of these large grains still present in the outer disk of CY Tau.

\subsection{Comparison with Other Disks}

Over the past several years there have been multiple examples of circumstellar disks where the interpretation of radial variations of the dust properties is supported by resolved observations at $\mathrm{mm}$ and $\mathrm{cm}$ wavelengths (Isella et al. 2010; Guilloteau et al. 2011; Trotta et al. 2013; Menu et al. 2014; Kwon et al. 2015). For example, in TW Hya, Menu et al. (2014) found that a radially homogeneous distribution of small and large grains (larger than $\sim 100 \mu \mathrm{m}$ ) was not a suitable representation of the observations, and a steeper $\Sigma(R)$ for the larger grains was required to fit these multi-wavelength dataset. And recently Kwon et al. (2015) studied a sample of disks and found that those with a steep midplane density gradient generally possess a smaller value of $\beta$, indicative of dust growth and radial evolution. The largest sample to date has been gathered by Guilloteau et al. (2011), where dual-wavelength observations of disks in Taurus-Auriga at angular resolutions between 0". 4 and 1 !" 0 were presented. This study found evidence for changes in the value of $\beta$ for several sources including CY Tau. Albeit limited by the angular resolution of the lowest frequency band, which corresponds to $\sim 100 \mathrm{AU}$ resolution for their $2.7 \mathrm{~mm}$ observations of CY Tau, these authors infer a change from $\beta \sim 0$ in the inner disk to high $\beta$-values in the outer disk of this young star. However, given the closeness in frequency of the two wavelengths studied by Guilloteau et al. (2011), together with the lack of high spatial resolution at $2.7 \mathrm{~mm}$, implies that their constraint is consistent with a constant value of $\beta$ at the $3 \sigma$ level for this particular object. As shown in Section 5.2, our increased wavelength coverage and high sensitivity allows us to discriminate radial variations of the dust emissivity index for CY Tau with high significance, where a constant value of $\beta$ is excluded at more than $7 \sigma$. This arises because the uncertainty in the spectral index $\left(\sigma_{\alpha}\right)$ is directly related to the wavelength coverage and SNR of the observations. In the case of dualwavelength observations, simple error propagation results in an uncertainty of $\sigma_{\alpha}^{2}=\left(\ln \left(\nu_{1} / \nu_{2}\right)\right)^{-2}\left(\mathrm{SNR}_{\nu_{1}}^{-2}+\mathrm{SNR}_{\nu_{2}}^{-2}\right)$, which indicates that an efficient way to reduce the uncertainty in $\alpha$, and thus in $\beta$, is to expand the wavelength coverage.

To compare radial changes in the dust properties for different circumstellar disks, we have gathered measurements from the literature that attempt to constrain $\beta(R)$, mostly from observations at the 1 and $3 \mathrm{~mm}$ bands. Since most of the available measurements barely resolve each circumstellar disk, we employ only two representative measurements of $\beta$, one from the inner disk regions and another from the outer disk. Thus, for each published result we compile the value of $\beta$ and its $1 \sigma$ uncertainty at the best resolution of the observations (between $\sim 20$ and $50 \mathrm{AU}$ depending on each target) to illustrate the inner disk constraint in $\beta$, and at either the observed outer disk radius or at $R=120 \mathrm{AU}$, whichever is smallest, to represent the constraint of $\beta$ in the outer disk (except for RY Tau and DG Tau, which at the outer disk radius have an unconstrained value of $\beta$; for these we select the outermost radii for which a $\beta$ constraint is still significant).

The compiled literature measurements are presented in the left panel of Figure 15, where we have computed the slope in $\beta$ 
$(R)$ between the inner and outer disk for each star (dashed lines), and the average slope in $\beta(R)$ for all the disks analyzed in the literature (gray shaded region). The steepest change in the dust emissivity spectral index occurs for DG Tau B (where $\beta=0.1 \pm 0.3$ at $20 \mathrm{AU}$, increasing to $\beta=1.5 \pm 0.3$ at 120 AU; Guilloteau et al. 2011). Excluding the added uncertainty in $\beta$ due to the absolute flux scale uncertainty for the two millimeter-wave bands observed for DG Tau B, the resulting change in $\beta(R)$ for this object is comparable to the constraints found for CY Tau, DoAr 25, and AS 209, which are presented together on the left panel of Figure 15.

Interestingly, the mean $\beta(R)$ profile from literature measurements is less steep than for the 3 disks analyzed in a consistent fashion. This could be due to the fact that most previously published observations only include dust emission traced at millimeter wavelengths ( 1 and $3 \mathrm{~mm}$ bands), which may be optically thick and thus reduce the overall value of $\beta$. Since the young stars presented in the left panel of Figure 15 boast some of the brightest disks observed in millimeter continuum emission, they represent a population of massive disks where high optical depth at millimeter wavelengths may be expected. Thus, including long-wavelength observations is critical for the calculation of $\beta(R)$, not only for the increased lever arm but also to avoid regions of high optical depth. We note that the comparison between previous results in the literature $\left(\beta_{\mathrm{mm}}(R)\right)$ and our multiwavelength analysis $\left(\beta_{\mathrm{mm}-\mathrm{cm}}(R)\right)$ should ideally be performed over a similar wavelength range to avoid potential biases between different wavelengths that trace different grain sizes, and could explain some of the differences between $\beta_{\mathrm{mm}}(R)$ and $\beta_{\mathrm{mm}-\mathrm{cm}}(R)$ seen in Figure 15 . Finally, we note that CY Tau shows the steepest rise in $\beta$ with radius, indicative of a substantial change in dust properties over tens of AU in this disk, while the smoothest rise in the emissivity spectral index occurs for AS 209 in the inner disk and for DoAr 25 in the outer disk. However, these differences at not significant when the disks are compared at the $3 \sigma$ level.

\section{CONCLUSIONS}

We present multi-wavelength observations of the protoplanetary disks surrounding the young stars CY Tau and DoAr 25. Observations at $0.9,1.3,2.8,8.0$, and $9.8 \mathrm{~mm}$ spatially resolve the dust continuum emission from these disks, down to scales of tens of AU. From observations obtained at $5 \mathrm{~cm}$, we quantify the amount of continuum emission whose origin is not thermal dust emission. We estimate the level of contamination for DoAr 25 to be $<24 \%$ of the integrated emission at 8.0 and $9.8 \mathrm{~mm}$, while for CY Tau we establish an upper limit of contamination of $<4 \%$ of the total emission at $7.1 \mathrm{~mm}$. Although these levels of contamination may seem small, they need to be quantified when characterizing the dust emission spectrum. The temperature and surface density profiles for both disks were constrained at all observed wavelengths shorter than $5 \mathrm{~cm}$, and from this modeling, we find that a constant dust opacity with radius does not fit these observations. This result is supported by the observational fact that the normalized visibility profiles of the dust continuum emission differ for different wavelengths: cm-wave observations trace emission from a compact disk structure, while mmwave observations trace a more extended disk structure.

Since our modeling indicates that the dust continuum emission is optically thin over the spatial scales explored (and this is also warranted by the observed brightness temperature profiles in Section 3.4), the observed emission spectrum will be directly proportional to the dust opacity spectrum, which allows us to infer radial variations of the dust opacity spectral index, $\beta(R)$. We find that a constant value of $\beta$ is excluded by our observations at more than $7 \sigma$ in both disks. Close to the central protostar, for $R<50 \mathrm{AU}$, the constraints on $\beta(R)$ are of high significance and indicate a rapid change of the dust properties between the inner disk and the outer disk. In the outermost regions of the disk where the dust emission has lower SNR, we can only set an lower limit for the dust opacity spectral index and we find that $\beta>1.0$ for CY Tau at $R>80$ AU, while $\beta>1.2$ for DoAr 25 at $R>80$ AU. We find that for the disks of AS 209, CY Tau, and DoAr 25, the profiles of $\beta(R)$ are steeper than for the available $\beta(R)$ constraints from the literatureat the $1 \sigma$ level (Isella et al. 2010; Guilloteau et al. 2011; Trotta et al. 2013), while these are all consistent at the $3 \sigma$ level. The increased wavelength coverage presented here and by Pérez et al. (2012) is critical for obtaining constraints of high significance on $\beta(R)$.

Assuming that the changes in the dust properties discussed above arise only from changes in the maximum particle size of the disk grain size distribution, we derive radial variations of the maximum grain size, $a_{\max }(R)$. For the two different grain size distribution explored, $q=2.5$ and $q=3.5$, we find a gradient in $a_{\max }$ with increasing grain size at smaller radial distance from the star, in both the CY Tau and DoAr 25 disks. We compare the observational constraints in $a_{\max }(R)$ with theoretical expectations of the maximum grain size when a population of grains grows and evolves while being limited by fragmentation or by radial drift. For an assumed disk turbulence of $\alpha_{t}=0.01$, the fragmentation barrier does not seem to be the limiting factor in the growth of these grains, as we observe millimeter and centimeter dust particles at distances from the star where the fragmentation barrier should have depleted these large particles. However, our observations seem to be consistent with a low-turbulence disk midplane with $\alpha_{t} \approx$ 0.001 in CY Tau and DoAr 25, as well as in AS 209 (Pérez et al. 2012). If disks are generally low-turbulence, then fragmentation may play a lesser role in shaping the grain size distribution of protoplanetary disks. Future constraints on disk turbulence will be necessary to assess this process. For the CY Tau disk, the population of grains inferred from our observations is above the growth barrier imposed by radial drift and fragmentation, with a difference of of a factor of a few at $\gtrsim 100 \mathrm{AU}$ radius. We hypothesize that this difference could be readily explained if a region of higher gas pressure exists in the outer disk, and is keeping these millimeter-sized particles from drifting inwards. Future high resolution observations that trace millimeter dust grains, as well as the gas distribution, are needed. Observations with the Atacama Large sub-Millimeter Array (ALMA) will be extremely well suited for such investigations, as spatial resolutions matching the VLA observations at $7 \mathrm{~mm}$ (of $0 . \prime 07$ or better) are already possible (ALMA Partnership et al. 2015). Finally, future observations of multiple disks at different stages of evolution (classical versus transitional), as well as different stellar mass or disk masses, will provide valuable information related to the first steps toward planet formation.

We thank the referee for valuable comments. The National Radio Astronomy Observatory is a facility of the National Science Foundation operated under cooperative agreement by 
Associated Universities, Inc. Ongoing CARMA development and operations are supported by the National Science Foundation under a cooperative agreement, and by the CARMA partner universities. The SMA is a joint project between the Smithsonian Astrophysical Observatory and the Academia Sinica Institute of Astronomy and Astrophysics, funded by the Smithsonian Institution and Academia Sinica. Part of this research was carried out at the Jet Propulsion Laboratory, Caltech, under a contract with the National Aeronautics and Space Administration.

Facilities:CARMA, SMA, VLA.

\section{REFERENCES}

ALMA Partnership, Brogan, C. L., Perez, L. M., et al. 2015, ApJL, 808, L3 Andrews, S. M., Chandler, C. J., Isella, A., et al. 2014, ApJ, 787, 148 Andrews, S. M., Hughes, A. M., Wilner, D. J., \& Qi, C. 2008, ApJL, 678, L133 Andrews, S. M., \& Williams, J. P. 2005, ApJ, 631, 1134

Andrews, S. M., \& Williams, J. P. 2007, ApJ, 671, 1800 Andrews, S. M., Wilner, D. J., Hughes, A. M., Qi, C., \& Dullemond, C. P. 2009, ApJ, 700, 1502

Asplund, M., Grevesse, N., Sauval, A. J., \& Scott, P. 2009, ARA\&A, 47, 481 Banzatti, A., Testi, L., Isella, A., et al. 2011, A\&A, 525, A12

Barge, P., \& Sommeria, J. 1995, A\&A, 295, L1

Beckwith, S. V. W., \& Sargent, A. I. 1991, ApJ, 381, 250

Bertout, C., Siess, L., \& Cabrit, S. 2007, A\&A, 473, L21

Birnstiel, T., Dullemond, C. P., \& Brauer, F. 2010, A\&A, 513, A79

Birnstiel, T., Klahr, H., \& Ercolano, B. 2012, A\&A, 539, A148

Blum, J., \& Wurm, G. 2008, ARA\&A, 46, 21

Boudet, N., Mutschke, H., Nayral, C., et al. 2005, ApJ, 633, 272

Calvet, N., D’Alessio, P., Hartmann, L., et al. 2002, ApJ, 568, 1008

Chiang, E. I., \& Goldreich, P. 1997, ApJ, 490, 368

Deckers, J., \& Teiser, J. 2013, ApJ, 769, 151

Dohnanyi, J. S. 1969, JGR, 74, 2531

Draine, B. T. 2006, ApJ, 636, 1114

Dullemond, C. P., \& Dominik, C. 2005, A\&A, 434, 971

Dullemond, C. P., Dominik, C., \& Natta, A. 2001, ApJ, 560, 957

Guilloteau, S., Dutrey, A., Piétu, V., \& Boehler, Y. 2011, A\&A, 529, A105

Guilloteau, S., Simon, M., Piétu, V., et al. 2014, A\&A, 567, A117

Hartmann, L., Calvet, N., Gullbring, E., \& D'Alessio, P. 1998, ApJ, 495, 385

Heißelmann, D., Blum, J., Fraser, H. J., \& Wolling, K. 2010, Icar, 206, 424

Henning, T., Michel, B., \& Stognienko, R. 1995, P\&SS, 43, 1333

Henning, T., \& Mutschke, H. 1997, A\&A, 327, 743

Henning, T., \& Stognienko, R. 1996, A\&A, 311, 291

Hill, C. R., Heißelmann, D., Blum, J., \& Fraser, H. J. 2015, A\&A, 573, A49

Isella, A., Carpenter, J. M., \& Sargent, A. I. 2009, ApJ, 701, 260

Isella, A., Carpenter, J. M., \& Sargent, A. I. 2010, ApJ, 714, 1746

Johansen, A., \& Klahr, H. 2005, ApJ, 634, 1353

Kataoka, A., Okuzumi, S., Tanaka, H., \& Nomura, H. 2014, A\&A, 568, AA42

Klahr, H. H., \& Henning, T. 1997, Icar, 128, 213

Krijt, S., Ormel, C. W., Dominik, C., \& Tielens, A. G. G. M. 2015, A\&A, 574, A 83

Kwon, W., Looney, L. W., Mundy, L. G., \& Welch, W. J. 2015, ApJ, 808, 102

Li, A., \& Draine, B. T. 2001, ApJ, 554, 778

Li, H., Finn, J. M., Lovelace, R. V. E., \& Colgate, S. A. 2000, ApJ, 533, 1023
Loinard, L., Torres, R. M., Mioduszewski, A. J., \& Rodríguez, L. F. 2008, ApJL, 675, L29

Lommen, D., Maddison, S. T., Wright, C. M., et al. 2009, A\&A, 495, 869

Lovelace, R. V. E., Li, H., Colgate, S. A., \& Nelson, A. F. 1999, ApJ, 513,805

Lynden-Bell, D., \& Pringle, J. E. 1974, MNRAS, 168, 603

Mamajek, E. E. 2008, AN, 329, 10

Mathis, J. S., Rumpl, W., \& Nordsieck, K. H. 1977, ApJ, 217, 425

McClure, M. K., Furlan, E., Manoj, P., et al. 2010, ApJS, 188, 75

Menu, J., van Boekel, R., Henning, T., et al. 2014, A\&A, 564, AA93

Miyake, K., \& Nakagawa, Y. 1993, Icar, 106, 20

Mundy, L. G., McMullin, J. P., Grossman, A. W., \& Sandell, G. 1993, Icar, 106,11

Natta, A., \& Testi, L. 2004, in ASP Conf. Ser. 323, Star Formation in the Interstellar Medium: In Honor of David Hollenbach ed. D. Johnstone et al. (San Francisco, CA: ASP), 279

Natta, A., Testi, L., Calvet, N., et al. 2007, in Protostars and Planets V, ed. B. Reipurth, D. Jewitt \& K. Keil (Tucson, AZ: Univ. Arizona Press), 767

Olofsson, J., Augereau, J.-C., van Dishoeck, E. F., et al. 2009, A\&A, 507, 327

Pascucci, I., Sterzik, M., Alexander, R. D., et al. 2011, ApJ, 736, 13

Pérez, L. M., Carpenter, J. M., Chandler, C. J., et al. 2012, ApJL, 760, L17

Pérez, L. M., Lamb, J. W., Woody, D. P., et al. 2010, ApJ, 724, 493

Pinilla, P., Benisty, M., \& Birnstiel, T. 2012a, A\&A, 545, AA81

Pinilla, P., Birnstiel, T., Ricci, L., et al. 2012b, A\&A, 538, AA114

Pollack, J. B., Hollenbach, D., Beckwith, S., et al. 1994, ApJ, 421, 615

Reynolds, S. P. 1986, ApJ, 304, 713

Ricci, L., Mann, R. K., Testi, L., et al. 2011, A\&A, 525, AA81

Ricci, L., Testi, L., Natta, A., \& Brooks, K. J. 2010a, A\&A, 521, A66

Ricci, L., Testi, L., Natta, A., et al. 2010b, A\&A, 512, A15

Ricci, L., Trotta, F., Testi, L., et al. 2012, A\&A, 540, AA6

Robitaille, T. P., Whitney, B. A., Indebetouw, R., \& Wood, K. 2007, ApJS, 169,328

Rodmann, J., Henning, T., Chandler, C. J., Mundy, L. G., \& Wilner, D. J. 2006, A\&A, 446, 211

Sault, R. J., Teuben, P. J., \& Wright, M. C. H. 1995, adass IV, 77, 433

Semenov, D., Henning, T., Helling, C., Ilgner, M., \& Sedlmayr, E. 2003, A\&A, 410,611

Shakura, N. I., \& Sunyaev, R. A. 1973, A\&A, 24, 337

Testi, L., Birnstiel, T., Ricci, L., et al. 2014, in Protostars and Planets VI, ed. H. Beuther, R. S. Klessen, C. P. Dullemond \& T. Henning (Tucson, AZ: Univ. Arizona Press), 339

Testi, L., Natta, A., Shepherd, D. S., \& Wilner, D. J. 2001, ApJ, 554, 1087

Testi, L., Natta, A., Shepherd, D. S., \& Wilner, D. J. 2003, A\&A, 403, 323

Torres, R. M., Loinard, L., Mioduszewski, A. J., \& Rodríguez, L. F. 2007, ApJ, 671,1813

Trotta, F., Testi, L., Natta, A., Isella, A., \& Ricci, L. 2013, A\&A, 558, AA64 Ubach, C., Maddison, S. T., Wright, C. M., et al. 2012, MNRAS, 425, 3137

Warren, S. G. 1984, ApOpt, 23, 1206

Weidenschilling, S. J. 1977, MNRAS, 180, 57

Williams, J. P., \& Best, W. M. J. 2014, ApJ, 788, 59

Wilner, D. J., D'Alessio, P., Calvet, N., Claussen, M. J., \& Hartmann, L. 2005, ApJL, 626, L109

Wilson, T. L., Rohlfs, K., \& Hüttemeister, S. 2009, in Tools of Radio Astronomy, ed. Thomas L. Wilson, K. Rohlfs \& S. Hüttemeister (Berlin: Springer-Verlag), 14

Youdin, A. N. 2010, EAS Publications Series, 41, 187

Zauderer, B. A., Bolatto, A. D., Vogel, S. N., et al. 2014, arXiv:1410.5560

Zubko, V. G., Mennella, V., Colangeli, L., \& Bussoletti, E. 1996, MNRAS, 282, 1321 